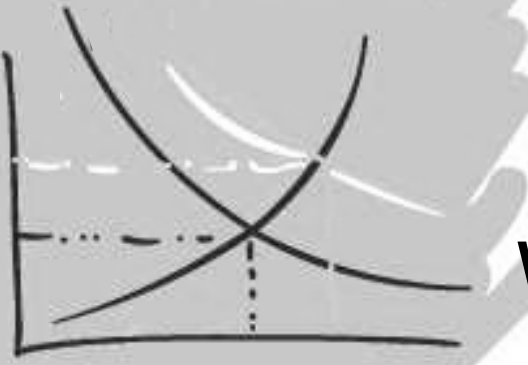

\title{
ECONOMIC ANALYSIS
}

WORKING PAPER SERIES

Estimación de los Flujos de Transporte de Mercancías

Interregionales Trimestrales mediante Técnicas de Interpolación Temporal

Nuria Gallego López, Carlos Llano Verduras, v J ulián Pérez García

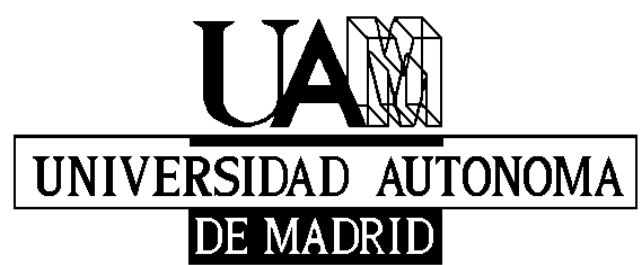

DePARTAMENTO DE ANÁlISIS ECONÓMICO: TEORÍA ECONÓMICA E HISTORIA ECONÓMICA 


\title{
Estimación de los flujos de transporte de mercancías interregionales trimestrales mediante técnicas de interpolación temporal $^{1}$
}

\author{
NURIA GALLEGO LÓPEZ \\ Personal Investigador en Formación del Departamento de Análisis Económico: Teoría e Historia \\ Económica de la Universidad Autónoma de Madrid. nuria.gallego@uam.es
}

CARLOS LLANO VERDURAS.

Profesor Contratado Doctor del Departamento de Análisis Económico: Teoría e Historia Económica de la Universidad Autónoma de Madrid.Email: carlos.llano@uam.es

JULIÁN PÉREZ GARCÍA.

Profesor Titular del Departamento de Economía Aplicada. Instituto L.R.Klein. CEPREDE. Universidad Autónoma de Madrid.julian.perez@uam.es

\section{RESUMEN:}

En el presente artículo se presenta la metodología y los resultados obtenidos en la trimestralización de los flujos de mercancías dentro de España. Partiendo de los datos anuales de movimientos de mercancías (1995-2007) según los principales modos de transporte disponibles en la base de datos C-intereg, se estiman los correspondientes vectores de movimientos de mercancías intra e interregionales de cada región con el Resto de España, en unidades físicas (toneladas) para el periodo 1995.I-2009.II. Para ello se utilizan métodos de interpolación y extrapolación temporal de carácter uni-variante Chow-Lin, (1971) y multivariante Denton, (1971); Di Fonzo, (1994), aplicados sobre indicadores de alta frecuencia relativos a los movimientos de mercancías en España. Los resultados obtenidos son analizados en comparación con otros indicadores trimestrales de referencia, y la capacidad de predicción del método es evaluada mediante el MAPE para el último año con datos observados (2007).

Palabras clave: comercio interregional, flujos de transporte, métodos de interpolación temporal, método chow-lin.

Clasificación JEL: C15, C22, C43, M40, R12, F17

\footnotetext{
1 La investigación que se recoge en este artículo se enmarca en el contexto del proyecto C-intereg (www.c-intereg.es ) y del programa TransporTrade S2007/HUM/497, financiado por la Consejería de Educación de la Comunidad de Madrid. www.uam.es/transportrade . Parte de esta investigación se relaciona con el Trabajo de Fin del Master de Economía Internacional de la UAM desarrollado por Nuria Gallego bajo la dirección de Carlos Llano. Los autores quieren agradecer los comentarios realizados por Maria Isabel García, Félix Muñoz y Jose Luís Zofío, durante la defensa de dicho trabajo. Igualmente se agradecen las sugerencias recibidas durante la presentación del artículo en el Congreso de Ciencia Regional celebrado en Valencia en Noviembre de 2009 .
} 


\section{Introducción}

En la mayor parte de los países existe más información sobre las relaciones comerciales que se mantienen con el extranjero que de las que realizan las propias regiones dentro del país. Este circunstancia contrasta con uno de los hechos estilizados más visitados de la reciente literatura sobre comercio internacional, tal y como es la presencia generalizada de importantes efectos frontera en la mayor parte de los países. Este fenómeno, descrito inicialmente por McCallum (1995), y desarrollado por diversos autores (Helliwell 1996; Anderson and van Wincoop 2003; Chen, 2004, Okubo, 2004 entre otros), constata que la mayor parte de los países mantienen relaciones comerciales mucho más intensas consigo mismos que con cualquier otro país de similares características y tamaño.

En el caso de España, aunque el mayor volumen del comercio interior ha quedado claramente constatado en todas las publicaciones existentes sobre la materia (Oliver et al, 1998; Pulido et al, 2002, 2001; Oliver et al, 2003, Llano, 2004a, 2004b; Minondo, 2003; Gil et al.; 2005; Requena y Llano, 2009), algunos trabajos recientes (Llano et al, 2009a, 2009b; Ghemawat et al, 2009) han podido detectar un lento pero progresivo cambio de patrón, según el cual, el peso relativo del comercio internacional se estaría incrementando en los últimos años, traduciéndose en un adelgazamiento del efecto frontera, al menos en las comunidades autónomas más abiertas y dinámicas. Con el paso del tiempo, este proceso podría alterar la situación actual de compenetración de las estructuras productivas regionales, con los consecuentes cambios en la evolución sincrónica de los ciclos regionales. Adicionalmente, cualquier cambio en la orientación del comercio y en la sustitución de los socios naturales de cada región (ellas mismas y el resto de regiones españolas por otros países más o menos lejanos) podría generar cambios en las vinculaciones productivas intersectoriales-interregionales, en los flujos fiscales y de renta que de ellos se derivan y en las necesidades de infraestructuras del transporte que hacen posibles dichos intercambios.

El carácter dinámico del proceso, y la importancia de sus posibles consecuencias, ponen de manifiesto la conveniencia de realizar un seguimiento más preciso de la evolución del comercio que cada comunidad autónoma mantiene con cada uno de los mercados (interior, resto de regiones y resto del mundo), no ya solo con un 
carácter anual sino incluso con una mayor periodicidad. Para ello es necesario disponer de indicadores de coyuntura adecuados, capaces de recoger la intensidad del comercio que cada comunidad mantiene con dichos mercados. En la actualidad, aunque se dispone de información coyuntural acerca del comercio internacional y de algunos indicadores de producción y consumo regional, resulta muy difícil determinar la procedencia geográfica de los tirones de demanda nacional, y delimitar qué parte de la evolución diferencial del crecimiento regional viene motivado por la evolución de la actividad intrarregional o del resto de regiones. Ante la inexistencia de encuestas trimestrales regionales sobre el destino de las ventas o el origen de las compras, y ante el elevado nivel de opacidad de la información fiscal existente acerca del comercio interregional, la principal fuente de información tiene que proceder necesariamente de los flujos de transporte de mercancías. Centrándonos en este tipo de información, los principales trabajos que han partido de los flujos de transporte de mercancías para estimar los flujos comerciales interregionales en España han puesto el énfasis en la estimación de los flujos anuales (Oliver et al, 2003, Llano, 2004a, 2004b; Llano et al, 2009). Por el contrario, los indicadores coyunturales sobre movimientos de mercancías son raramente incorporados en los análisis de coyuntura regionales, quedando relegados a publicaciones específicas del sector del transporte (ver por ejemplo, el Anuario estadístico del Ministerio de Fomento, o los informes periódicos de Puertos del Estado).

Con el objeto de avanzar en el conocimiento del comercio interior en el marco coyuntural, el presente artículo plantea una posible metodología para la estimación del comercio intra e interregional de bienes a nivel trimestral en España. Más concretamente, en este artículo nos centramos específicamente en la estimación de los flujos de transporte de mercancías en unidades físicas (toneladas), dejando para futuros trabajos la trimestralización de los flujos en valor. Para ello, tomando como referencia los datos anuales sobre transporte de mercancías con carácter intrarregional e interregional de la base de datos del proyecto C-intereg (www.c-intereg.es), se han estimado los correspondientes vectores trimestrales del comercio intrarregional e interregional de bienes de cada región con el resto de España. La metodología utilizada entronca con los trabajos previos sobre interpolación temporal y estimación de magnitudes trimestrales, haciendo uso de algunos de los métodos de interpolación 
temporal uni-variantes (Chow-Lin, 1971) y multi-variantes (Denton, 1971, di Fonzo, 1994) más habituales en la literatura.

La estructura del trabajo es la siguiente: en la sección 1, se explica la metodología utilizada en la estimación trimestral, haciendo una breve síntesis de los métodos estadísticos de interpolación temporal basados en indicadores, prestando una especial atención a los métodos de Chow-Lin y Denton. La sección 2 describe el proceso aplicado a la trimestralización de los flujos de transporte en unidades físicas (en Tm.). Posteriormente, en la Sección 3, se analizan los primeros resultados de la estimación y de la predicción, valorando la capacidad predictiva de los modelos utilizados. Finalmente, el documento termina con las correspondientes conclusiones y referencias bibliográficas.

\section{Métodos estadísticos de interpolación temporal ${ }^{2}$}

Son muchos los modelos que se han formulado para obtener una serie de alta frecuencia a partir de otra de baja frecuencia. En la amplia literatura disponible sobre métodos de interpolación temporal (Sanz, 1991; Matea y Regil, 1994; Cavero et al, 1994, OCDE, 1996; Pons et al, 1997; Cabrer y Pavía, 1999; Pavía, 2000; Quilis 2001a y 2001b; Pavía y Borrás, 2007; Pavía et al, 2003a y 2003b; Pavía-Miralles y CabrerBorras, 2007, entre otros), se describen procedimientos que van desde la pura extrapolación lineal hasta otros más complejos basados en indicadores y que permiten la satisfacción simultánea de múltiples restricciones temporales, sectoriales y territoriales. Por un lado, los métodos de interpolación temporal suelen clasificarse según la naturaleza más o menos automática del procedimiento y la utilización de información económica como referencia. Desde este punto de vista, se suele diferenciar entre métodos basados en técnicas estadísticas (Boot et al, 1967) y métodos basado en la utilización de indicadores económicos. Así mismo, los métodos pueden ser "univariantes" o "multi-variantes" según contemplen la trimestralización individual o simultánea de la variable objetivo: por el lado de los métodos uni-variantes (Boot et al.,

\footnotetext{
${ }^{2}$ Esta sección describe la formulación matemática de los métodos de interpolación utilizados. La sección está inspirada en algunos trabajos como: Quilis (2001), Pavía et al. (2003).
} 
1967, Fernández, 1981, Chow-Lin, 1971, Litterman, 1983, Santos y Cardoso, 2001) el objetivo sería la estimación independiente de cada una de las series trimestrales compatibles con la misma serie en periodicidad anual, mientras que por el lado de los métodos multi-variantes (Denton, 1971, Rossi, 1982, Di Fonzo 1990; Pavía y Cabrer, 2007), se buscaría la estimación simultánea de un conjunto de series trimestrales capaces de cumplir al mismo tiempo dos tipos de restricciones, una longitudinal y otra transversal.

Desde un punto de vista intuitivo, la estimación basada en indicadores plantea la estimación en alta frecuencia de la magnitud objetivo (dato trimestral que se quiere estimar), a partir de la evolución de un indicador (o conjunto de indicadores) disponible en alta frecuencia y que presentan una alta correlación con la magnitud objetivo en términos agregados (datos anuales conocidos). Con ello, se está asumiendo que los indicadores que tienen una evolución similar a la de la variable objetivo en términos anuales, también lo tendrán en términos trimestrales. Junto al problema de selección de los indicadores, los métodos de interpolación deben asegurar que la magnitud objetivo estimada en alta frecuencia sea compatible (por agregación suma o media, por ejemplo), con los datos conocidos de la magnitud objetivo disponible en términos anuales. Por otro lado, será necesario asumir una serie de hipótesis acerca del comportamiento y reparto del término de error, pudiendo ser éste puramente aleatorio o venir determinado por una estructura estocástica que recoja el comportamiento no explicado por los indicadores.

De una manera más formal, la relación entre la variable objetivo y los indicadores en alta frecuencia puede ser expresada de la siguiente manera:

$y_{t}=\beta x_{t}+u_{t}$

Donde $y_{t}$ se refiere a la variable objetivo en alta frecuencia, en nuestro caso trimestral, no observable, $\beta$ representa un vector de parámetros desconocidos, $\mathrm{x}_{\mathrm{t}}$ corresponde a los indicadores observables en alta frecuencia y donde $u_{t}$ supone una perturbación cuya estructura puede asumir múltiples formas.

A partir de aquí, los distintos métodos uni-variantes de interpolación tratarán de estimar la variable objetivo en alta frecuencia, $\mathrm{y}=\left(\mathrm{y}_{1}, \ldots, \mathrm{y}_{\mathrm{n}}\right)$, partiendo de la propia 
variable objetivo en baja, $\mathrm{Y}=\left(\mathrm{Y}_{1}, \ldots, \mathrm{Y}_{\mathrm{N}}\right)$, y de los $p$ indicadores $\mathrm{x}=\left(\mathrm{x}_{1}, \ldots, \mathrm{x}_{\mathrm{n}}\right)$ de dimensión $n=4 N$, donde $n$ es el número total de trimestres y $N$ el número de años, verificando la restricción longitudinal y gozando de buenas propiedades. Cuando se impone el cumplimiento de la restricción longitudinal (ecuación 2), se hace referencia al objetivo de garantizar que la suma, o promedio, de los cuatro trimestres de un mismo año coincida con el valor anual de la serie objeto.

$$
\sum_{t-1}^{n} \hat{y}_{t, T}=Y_{T}
$$

Con los métodos multi-variantes de interpolación se podría añadir una restricción adicional a la longitudinal, ya que ésta únicamente garantiza que el flujo comercial trimestral de cada comunidad autónoma coincida con su agregado anual, sin considerar la interacción que pueda existir entre éstas. Mediante el enfoque multivariante se estaría hablando de añadir a la estimación una restricción transversal, con la que se consigue controlar el agregado trimestral de las distintas variables objetivo. En nuestro trabajo, esto supone que las exportaciones trimestrales interregionales deben coincir con las importaciones interregionales.

\subsection{El método uni-variante de Chow-Lin}

El método de interpolación propuesto por Chow-Lin (1971), toma como referencia el supuesto de que se puede formular un modelo en alta frecuencia que recoge la relación entre la matriz de $p$ indicadores y la serie trimestral inobservable (ecuación 1), sujeta a la restricción longitudinal (ecuación 2). De tal forma que, mediante la agregación de los indicadores, través de la premultiplicación de la matriz de agregación temporal C (ecuación 3), se obtiene el modelo anual (ecuación 4), que sí puede ser estimado.

$$
C=I_{N} \otimes c=\left[\begin{array}{ccccccccccccc}
1 & 1 & 1 & 1 & 0 & 0 & 0 & 0 & \ldots & 0 & 0 & 0 & 0 \\
0 & 0 & 0 & 0 & 1 & 1 & 1 & 1 & \ldots & 0 & 0 & 0 & 0 \\
\ldots & \ldots & \ldots & \ldots & \ldots & \ldots & \ldots & \ldots & \ldots & \ldots & \ldots & \ldots & \ldots \\
0 & 0 & 0 & 0 & 0 & 0 & 0 & 0 & \ldots & 1 & 1 & 1 & 1
\end{array}\right]
$$


La matriz de agregación temporal $\mathbf{C}$ permite la transformación de una serie de alta frecuencia en otra de baja. Donde $\mathrm{I}_{\mathrm{N}}$ es la matriz identidad, $\mathrm{N}$ representa el número de años que recoge la variable objetivo, ${ }^{\otimes}$ se refiere al producto tensorial de Kronecker y $\mathrm{c}=[1,1,1,1]$ es el vector de agregación por suma.

Una vez agregado el modelo inicial, nos encontramos ahora con un modelo en que se relaciona la serie anual $\mathrm{Y}$ con el indicador agregado $\mathrm{X}$ :

$Y=X \beta+U$

En el caso de que el término de error $U$ no sea ruido blanco, la estimación se deberá realizar por máxima verosimilitud (MV) o por mínimos cuadrados generalizados (MCG).

Donde la matriz de varianzas y covarianzas asociada al término de error tiene la forma:

$V=C v C^{\prime}$

Siendo $v$ la matriz de varianzas y covarianzas de la perturbación trimestral. Será precisamente la estructura de esta matriz $v$ la que marcará la diferencia básica entre los distintos métodos de trimestralización univariante que habitualmente se utilizan en la literatura. Chow-Lin, por ejemplo, supuso que ésta respondía a un proceso propio de un autorregresivo de orden uno. Igualmente sugirió la posibilidad de encontrarnos ante una perturbación ruido blanco de media cero y varianza constante.

Formalmente, la consideración del error como un autorregresivo de orden uno, determinará que la perturbación $u$ tome la siguiente forma:

$u_{t}=\theta u_{t-1}+a_{t} \quad$ donde $|\theta|<1 \quad \forall t$

Siendo la matriz de varianzas y covarianzas del error trimestral:

$v=\frac{\sigma^{2}}{\left(1-\rho^{2}\right)} v(\rho)$

Donde $v(\rho)$ es una matriz con la forma: 
$v(\rho)=\left[\begin{array}{cccc}1 & \rho & \ldots & \rho^{n-1} \\ \rho & 1 & \ldots & \rho^{n-2} \\ \ldots & \ldots & \ldots & \ldots \\ \rho^{n-1} & \rho^{n-2} & \ldots & 1\end{array}\right]$

En la estimación de los parámetros, Chow-Lin propuso un estimador lineal, insesgado y óptimo, de varianza mínima (ELIO), que satisface la restricción longitudinal [2] y que a la vez es compatible con el modelo de alta frecuencia que se estableció inicialmente [1]. El estimador lineal que cumple estas condiciones es de la siguiente forma:

$\hat{y}=A Y$

Donde A es la matriz que determina el estimador que debe cumplir las propiedades de insesgadez y varianza mínima esenciales, siendo la primera de ellas para generar la estructura básica del estimador y la segunda para lograr una estimación precisa.

\subsection{El método multivariante de Denton}

El método de interpolación de Denton para el caso multivariante (descrito en Denton, 1971) se encuentra diseñado para la descomposición simultánea de varias series temporales, permitiendo incluir una restricción adicional a la longitudinal [2], que hace referencia a la relación trasversal de las series. Concretamente, dicha restricción permite que la agregación trasversal de los resultados para las distintas series tome un valor especificado por el investigador. Esta metodología es habitual en la estimación de valores cuya coherencia debe cumplir una relación contable, como es en nuestro trabajo el hecho de que la suma de las importaciones interregionales trimestrales deba coincidir con la de las exportaciones interregionales.

La metodología desarrollada por Denton tiene como soporte la existencia de un vector en alta frecuencia de la forma $Z_{t}=\left[z_{1}, z_{2}, \ldots, z_{n}\right]$, constituido por datos reales o impuestos por el investigador. Dicho vector, supone la restricción transversal a la que está sujeta la estimación (ecuación 10), sin dejar de lado la restricción longitudinal (ecuación 2), con la que se consigue que la estimación en términos anuales coincida con 
la serie original. En resumen, el problema de base de esta metodología es la obtención de una series de nuevos vectores trimestrales $\mathbf{y}_{\mathbf{t}, \mathbf{j}}$ que cumplan las dos restricciones. Este hecho se traduce en que: por un lado, una vez agregados los $\mathbf{J}$ vectores estimados en un único vector $\mathbf{y}_{\mathbf{t}, \mathbf{J}}$, que contiene las $\mathbf{J}$ series de forma trimestral, éste muestre la mínima discrepancia posible con el vector $\mathrm{Z}_{\mathrm{t}}$ que hemos impuesto; así mismo, la estimación individualizada para cada serie debe satisfacer la restricción longitudinal.

$\sum_{j-1}^{J} \hat{y}_{j, t, T}=Z_{t, T}$

Donde $j$ muestra el número total de variables que se interpolan conjuntamente y $Z_{t, T}$ es el valor de la restricción para el trimestre $t$, año T.

\section{Metodología de estimación de los flujos trimestrales de transporte de mercancías}

Partiendo de las experiencias nacionales e internacionales de trimestralización de series regionales anuales, en este trabajo se procede a la trimestralización de los flujos de mercancías que se han producido en España a partir de 1995, y que han sido estimados previamente en el marco del Proyecto C-intereg (ver el Anexo para una breve descripción de las características de dicha base de datos). Por motivos de disponibilidad de datos y mayor eficiencia de las técnicas estadísticas utilizadas, el trabajo se centra en la trimestralización de los flujos intrarregionales e interregionales totales al nivel de comunidad autónoma (cada comunidad frente al resto de España), sin que por el momento se puedan disponer de los flujos trimestrales bilaterales, por tipos de productos o modos de transporte. Como ya se ha comentado en la introducción, en este trabajo nos centramos en un análisis detallado de los movimientos de mercancías en unidades físicas (Tm.). El ejercicio de trimestralización se ha centrado en el periodo 1995-2007 (para el que existen datos anuales sobre comercio intra e interregional para cada una de las comunidades) y ha incluido un ejercicio de predicción para el periodo 2008.I-2009.II (periodo máximo para el que se dispone de indicadores de alta frecuencia). 
En el comercio interregional, la suma de lo que se exporta es igual a la de lo que se importa. Dado que la estimación por métodos uni-variantes sólo asegura el cumplimiento de la restricción longitudinal (que la agregación de los trimestres coincida con el dato anual), ha sido necesaria la aplicación de métodos multi-variantes (Denton., $1971^{3}$ ) para asegurar que la suma de las exportaciones interregionales trimestrales estimadas coincidan con las importaciones interregionales trimestrales totales. Esta condición, que cumplen los datos anuales, debe cumplirse trimestralmente, tanto en el periodo de interpolación (1995.I-2007.IV) como de extrapolación (2008.I-2009.II).

Por estos motivos hemos optado por dividir el proceso en dos fases:

1) Primera fase: se realiza la estimación trimestral de los tres tipos de flujos de transporte de mercancías (intrarregionales e interregionales), bajo una metodología uni-variante (Chow-Lin) para el periodo completo 1995.I2007.IV, con una predicción uni-variante para el periodo 2008.I-2009.IV. En esta fase se obtiene la estimación final del flujo de exportaciones interregionales y del flujo intrarregional, ambos en Tm.

2) Segunda fase: mediante el método multivariante de Denton se han estimado las importaciones interregionales trimestrales definitivas, utilizando como indicadores las importaciones interregionales obtenidas en la etapa anterior. La restricción transversal empleada ha sido el valor trimestral nacional de las exportaciones interregionales, es decir, la suma de las exportaciones interregionales de las comunidades autónomas para cada trimestre obtenidas en el paso anterior. Mediante este procedimiento, se obtienen unas nuevas importaciones trimestrales para cada comunidad, que cumplen la restricción longitudinal a la vez que aseguran que su compatibilidad transversal con las exportaciones interregionales totales.

\footnotetext{
${ }^{3}$ La elección del método multivariante ha estado condicionado por varios aspectos. Por un lado, di Fonzo permite realizar predicciones y asegurar el cuadre longitudinal y transversal. Sin embargo, su aplicación a nuestros datos ha arrojado resultados inconexos, dada la dificultad de lograr la convergencia en paneles de datos donde la amplitud de los elementos de corte transversal superan a los años disponibles. Por el contrario, el método Denton no mostraba esta última limitación, aunque no permitía la posibilidad de predecir.
} 
Tabla 1: Etapas del proceso de estimación del comercio trimestral de bienes

Fase I: interpolación y extrapolación de las exportaciones, importaciones y comercio intrarregional en Tm. mediante técnicas uni-variantes (Chow-Lin)

a) Selección de los indicadores trimestrales para cada Comunidad Autónoma.

b) Análisis de los residuos anuales.

c) Estimación de las series trimestrales.

Fase II: interpolación y extrapolación de las importaciones interregional en Tm. mediante técnicas multi-variantes (Denton)

Partiendo de la visión sintética descrita en la Tabla 1, pasamos a analizar en detalle cada una de los pasos seguidos en la estimación de las series trimestrales de toneladas.

\subsection{Selección de indicadores sobre transporte de mercancías para cada comunidad}

Las técnicas de interpolación temporal descritas en el apartado 2, exigen que los indicadores compartan una evolución temporal similar a la del flujo que se quiere trimestralizar. Este hecho va a determinar la selección de indicadores, ya que habrá que descartar variables con escasa variabilidad temporal cómo son todas las variables estructurales que habitualmente caracterizan el comercio de una región (población, nivel de producción y empleo, stock de infraestructuras, accesibilidad, etc). Así mismo, el indicador deberá estar en la frecuencia a la que se pretende transformar la variable anual. Por último, tal y como se ha indicado en la introducción, uno de los objetivos de este trabajo es el de utilizar indicadores novedosos que estén relacionados con los flujos de transporte y la logística interna de las mercancías de producción y consumo interior.

Con estas exigencias, se han buscado indicadores que pudieran tener un comportamiento parecido al de la serie anual ${ }^{4}$ y estuvieran relacionadas con el movimiento de mercancías. En este sentido, lo deseable sería poder disponer de un conjunto amplio de indicadores capaces de explicar la evolución diferencial de los

\footnotetext{
${ }^{4}$ La elección del indicador para cada comunidad se realiza de acuerdo a su comportamiento respecto a la variable anual, medido por su correlación en términos anuales. La agregación de las toneladas necesaria para calcular las correlaciones de los indicadores frente al flujo se realiza mediante suma de los datos trimestrales.
} 
distintos modos de transporte utilizados en los desplazamientos interiores, con el objeto de atender a las situaciones específicas de cada región según su ubicación geográfica y especialización sectorial (Ej: regiones costeras, interiores o insulares; con o sin conexión aérea; con o sin presencia de sectores específicos, etc.). Así mismo, dado que el objetivo de la trimestralización contempla tres vectores para cada comunidad autónoma (exportaciones interregionales, importaciones interregionales y comercio intrarregional), es necesario buscar indicadores adecuados para cada uno de ellos.

Después de una profunda búsqueda de indicadores, y tras el análisis de las correlaciones entre los indicadores disponibles y la variable objetivo, nuestra atención se ha centrado principalmente en aquéllos que hacen referencia al volumen de mercancías transportadas por carretera de la Encuesta Permanente de Transporte de Mercancías por Carretera (EPTMC) del Ministerio de Fomento:

- Por un lado, el Ministerio de Fomento publica el volumen de mercancía movido intra e interregionalmente (cifras totales, no bilaterales), por carretera, con origen y destino nacional, para las comunidades de mayor tamaño" (Andalucía, Aragón, Castilla y León, Castilla la Mancha, Cataluña, Comunidad Valenciana, Comunidad de Madrid, Región de Murcia y País Vasco).

- Para el resto de comunidades autónomas (Asturias, Baleares, Canarias, Cantabria, Extremadura, Galicia, Navarra y La Rioja), se dispone del indicador de "Tráfico" (procedente también de la EPTMC) que recoge el volumen de mercancías (miles de Tm.) transportadas por carretera con destino nacional, sin separar entre flujos intra e interregionales de exportación e importación. Como consecuencia, en estos casos ha sido necesario separar el tráfico trimestral en flujos de salida, o entrada, asumiendo para ello las cuotas correspondientes a cada comunidad y año procedentes de los datos anuales de $\mathrm{C}$-intereg.

En el caso de Canarias los indicadores han sido los flujos de comercio interregional publicados por la Agencia Española de Administración Tributaria (AEAT) acerca de los flujos comerciales entre las islas Canarias y la Península Ibérica. En relación a Baleares, donde no existe una información equivalente a la de Canarias, se han buscado indicadores alternativos al del movimiento por carretera con el objeto de modelizar los flujos interregionales (exportaciones e importaciones). Tras probar con 
indicadores sobre flujos trimestrales de mercancías por avión, se ha comprobado que el mejor indicador disponible es el de cargas y descargas del transporte de mercancías por vía marítima para las operaciones de cabotaje recogidas por la Autoridad Portuaria de Baleares (datos facilitados por Puertos del Estado). Para una mayor claridad, la Tabla 2 recoge los indicadores utilizados para cada una de las comunidades autónomas y tipo de flujo.

Tabla 2. Indicadores utilizados para trimestralizar los flujos en Tm.

\begin{tabular}{|c|c|c|c|}
\hline \multirow{2}{*}{ Comunidad Autónoma } & \multicolumn{3}{|c|}{ Tipo de flujo(Tm.) } \\
\hline & Exportaciones & Importaciones & Intrarregional \\
\hline Andalucía & \multirow{9}{*}{ EPTMC } & \multirow{9}{*}{ EPTMC } & \multirow{9}{*}{ EPTMC } \\
\hline Aragón & & & \\
\hline Castilla y León & & & \\
\hline Castilla-La Mancha & & & \\
\hline Cataluña & & & \\
\hline C. Valenciana & & & \\
\hline Madrid & & & \\
\hline Murcia & & & \\
\hline País Vasco & & & \\
\hline Asturias & \multirow{6}{*}{$\begin{array}{c}\text { Trafico (EPTMC) } \\
\text { Estimación } \\
\text { Propia }\end{array}$} & \multirow{6}{*}{$\begin{array}{c}\text { Trafico (EPTMC) } \\
\text { Estimación } \\
\text { Propia }\end{array}$} & \multirow{6}{*}{$\begin{array}{c}\text { Trafico } \\
\text { (EPTMC) }\end{array}$} \\
\hline Cantabria & & & \\
\hline Extremadura & & & \\
\hline Galicia & & & \\
\hline Navarra & & & \\
\hline La Rioja & & & \\
\hline Baleares & $\begin{array}{c}\text { Puertos del } \\
\text { Estado }\end{array}$ & $\begin{array}{c}\text { Puertos del } \\
\text { Estado }\end{array}$ & Tráfico \\
\hline Canarias & AEAT-ISTAC & AEAT-ISTAC & Tráfico \\
\hline \multicolumn{4}{|c|}{$\begin{array}{l}\text { Fuente: Elaboración propia a partir de las siguientes fuentes: Cargas y Descargas } \\
\text { trimestrales en Tm. Para los flujos de Tráfico ha sido necesaria la estimación de } \\
\text { los flujos de carga y descarga a partir del dato agregado. Ambos indicadores } \\
\text { proceden de la EPTMC y son recogidos por el Boletín Estadístico del Ministerio } \\
\text { de Fomento. }\end{array}$} \\
\hline
\end{tabular}




\subsection{Análisis de los residuos 5}

Una vez verificada la alta correlación entre los indicadores y las variables objetivo en todas las regiones, se han estimado las regresiones por $\mathrm{MCO}$, dando paso al análisis residual y a la comprobación de la eficiencia e insesgadez de dicho estimador. Ante un residuo ruido blanco, bastaría utilizar MCO. La trimestralización se logra aplicando los coeficientes estimados en el modelo anual a los datos del indicador en alta frecuencia más un reparto igualitario del residuo entre las estimaciones. Si por el contrario los residuos muestran autocorrelación, los estimadores MCO dejarían de ser eficientes, debiéndose utilizar MCG o MV. En este caso el reparto trimestral del residuo anual se hará de acuerdo a la estructura específica de la matriz de varianzas y covarianzas del modelo en alta frecuencia.

De una forma global, se ha analizado la bondad del ajuste de las regresiones entre la variable endógena y los indicadores mediante los coeficientes de determinación (Tabla 8). Así mismo, para el estudio de los residuos se ha tomando como criterio el test de Durbin Watson. Adicionalmente, y para reforzar la fiabilidad de las conclusiones, se han utilizado los correlogramas de los residuos en todos los casos en los que el test ha caído en las zonas de indeterminación o en la de no aceptación de la hipótesis nula de no autocorrelación. Los resultados obtenidos en ambos análisis muestran que en todos los casos los residuos siguen un proceso ruido blanco, excepto en las exportaciones interregionales de Canarias, cuyo comportamiento es propio de un proceso autorregresivo de orden uno (ver resultados en la Tabla 9 del Anexo).

\subsection{Estimación de las series trimestrales mediante los métodos de interpolación}

Una vez determinado el comportamiento de los residuos, nos encontramos en disposición de poder aplicar la metodología más idónea de acuerdo a los distintos tipos

\footnotetext{
5 Desde el punto de vista empírico, la aplicación de las técnicas aquí descritas al problema específico de la trimestralización de los flujos interregionales de mercancías se ha realizado a través de las rutinas programadas por Abad y Quilis (2004), y que están disponibles en la red para su uso (www.spatial-econometrics.com ). En concreto, se ha trabajado con aquellas que permiten aplicar el método de Chow-Lin con el parámetro $\rho$ fijo y optimizado, tanto usando estimaciones basadas en Mínimos Cuadrados Ordinarios (MCO) como Mínimos Cuadrados Generalizados (MCG) y Máxima Verosimilitud (MV). Para la aplicación de la técnica multi-variante de Denton se han utilizado las rutinas correspondientes de los autores.
} 
de procesos. En el caso de las exportaciones interregionales, para aquellos casos en los que el residuo anual se comportaba según un proceso ruido blanco, se ha aplicado la metodología de Chow-Lin con parámetro $\rho=0$. Dicho parámetro representa la autocorrelación de los residuos, de tal forma que el reparto del residuo se divide por igual entre los cuatro trimestres de cada año. Para el caso de Canarias, se ha utilizado el modelo Chow-Lin con ajuste automático del parámetro $\rho$, de tal manera que es el propio modelo el que estima este parámetro de acuerdo con los datos. En el caso de los flujos intrarregionales, Canarias ha requerido de un trato especial, ante la presencia de un comportamiento atípico desde 2005 para ajustar la modelización a la presencia de un posible cambio estructural, introduciéndose una dummy que toma valor 1 para todos los trimestres del periodo 2005-2009, y cero en el resto de los casos.

\section{Análisis de resultados}

En este apartado se analizan los resultados obtenidos en la trimestralización de los flujos de transporte de mercancías anuales de la base de datos C-Intereg para el periodo 1995.I-2009.II. Por motivos de espacio, el análisis se centra en los datos agregados a nivel nacional ${ }^{6}$, dejando para trabajos posteriores un estudio pormenorizado de cada comunidad autónoma. Este análisis, se ha dividido en varios apartados: en primer lugar, se analizan los resultados obtenidos en la trimestralización de los flujos en unidades físicas y se comparan con la evolución de otras variables económicas de referencia como el VAB relativo a los sectores productores de bienes, el Índice de Producción Industrial (IPI) o el comercio internacional en toneladas (tanto el dato del VAB como del IPI se han obtenido del INE). A continuación, se analizan los resultados de la predicción y la bondad de la misma.

\footnotetext{
${ }^{6}$ Nótese que la suma de exportaciones interregionales en cada trimestre coincide con el de las importaciones interregionales
} 


\subsection{Análisis de las series trimestrales obtenidas}

Tanto en el Gráfico 1, como en la Tabla 3, comparamos los resultados obtenidos en la trimestralización de los flujos de transporte en Tm., tomando como referencia el VAB nacional trimestral corriente a precios de mercado de los sectores de agricultura e industria ${ }^{7}$. Pese a las diferencias existentes en las unidades de medida, podemos observar como las series comparten un cierto comportamiento estacional. Así mismo, el análisis simple contenido en la Tabla 3 muestra una alta correlación entre nuestras series y el VAB utilizado. Finalmente, queremos llamar la atención sobre el hecho de que los datos de predicción de nuestras series (2008.I-2009.II) han sido capaces de recoger los primeros meses de recesión experimentados por la economía española, y también recogidos por el VAB.

La elevada magnitud de los flujos intrarregionales en comparación a los interregionales (en Tm.) viene explicada por la tendencia a encontrar un gran volumen de movimientos de mercancías en un reducido radio de distancia. Este hecho, constatado periódicamente por la EPTMC (Ministerio de Fomento), está muy condicionado por la diferente composición sectorial de los flujos intrarregionales e interregionales, y la fuerte concentración de mercancías pesadas y de bajo valor económico (construcción y energía) en los flujos intrarregionales.

\footnotetext{
${ }^{7}$ Ante la ausencia de una serie oficial completa de VAB en términos constantes, se toma como referencia el VAB corriente.
} 
Gráfico 1: Evolución trimestral de las exportaciones interregionales (miles de Tm.), y el VAB corriente (millones de euros) de agricultura e industria. Periodo 1995.I-2009.II.

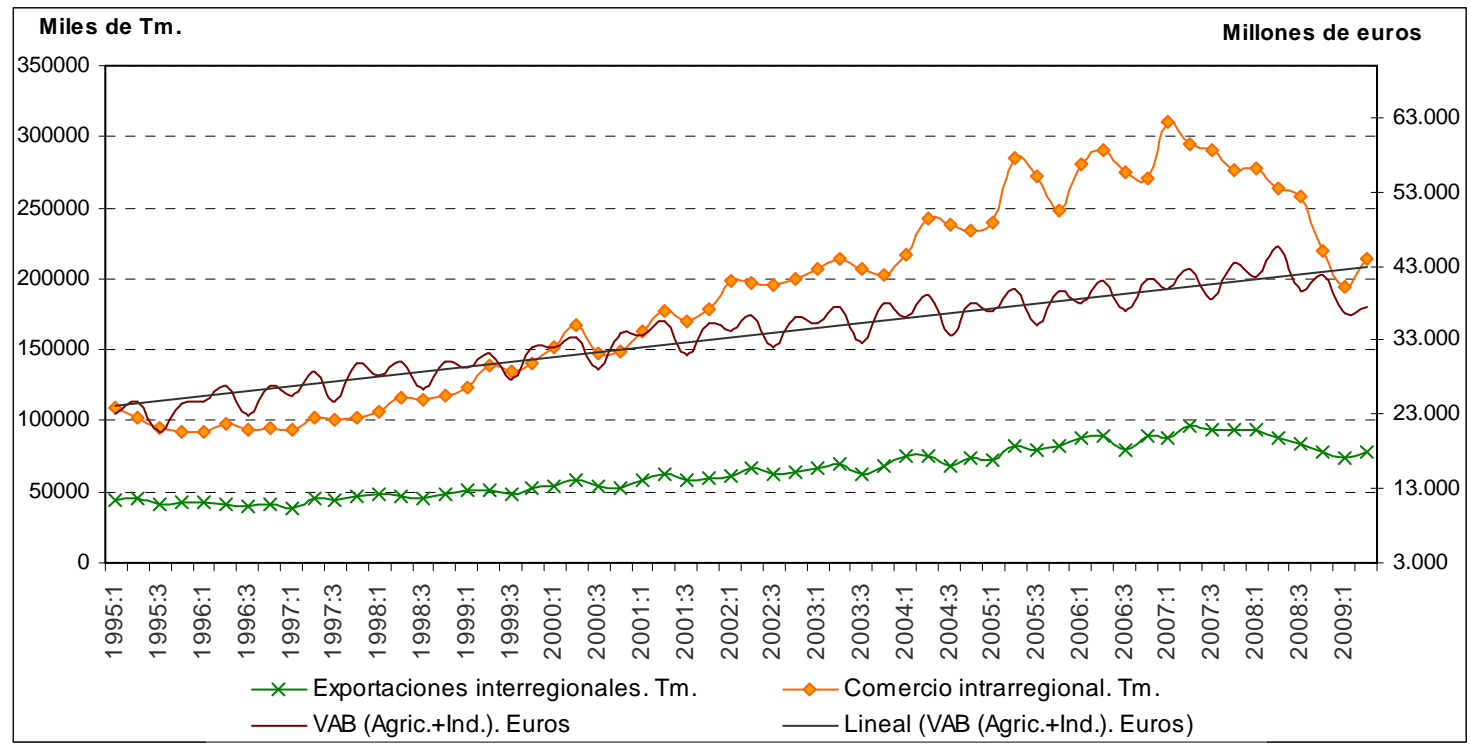

Fuente: Elaboración propia a partir de datos del INE.

Tabla 3. Matriz de correlaciones entre los vectores de comercio intrarregional e interregional total (en Toneladas) con el VAB corriente de agricultura e industria. 1995.I2009.II.

\begin{tabular}{|c|c|c|c|}
\cline { 2 - 4 } \multicolumn{1}{c|}{} & Exportaciones Tm. & $\begin{array}{c}\text { Comercio } \\
\text { intrarregional Tm. }\end{array}$ & $\begin{array}{c}\text { Valor añadido } \\
\text { bruto } €\end{array}$ \\
\hline Exportaciones Tm. & 1 & 0,98 & 0,93 \\
\hline $\begin{array}{c}\text { Comercio } \\
\text { intrarregional Tm. }\end{array}$ & 1 & 0,91 \\
\hline Valor añadido bruto $€$ & & & 1 \\
\hline \multicolumn{2}{|l|}{ Fuente: elaboración propia a partir de los flujos correspondientes. } \\
\hline
\end{tabular}

Para aportar un punto de referencia adicional no afectado por diferencias en las unidades de medida, se procede a comparar la evolución del comercio interior en Tm. (intrarregional más interregional) con el Índice Producción Industrial (IPI), uno de los índices clásicos de medición de la actividad industrial no afectado por la evolución de los precios. 
Gráfico 2 Evolución interanual del comercio interior (exportaciones interregionales + comercio intrarregional) y del IPI. 1995.I-2009.II

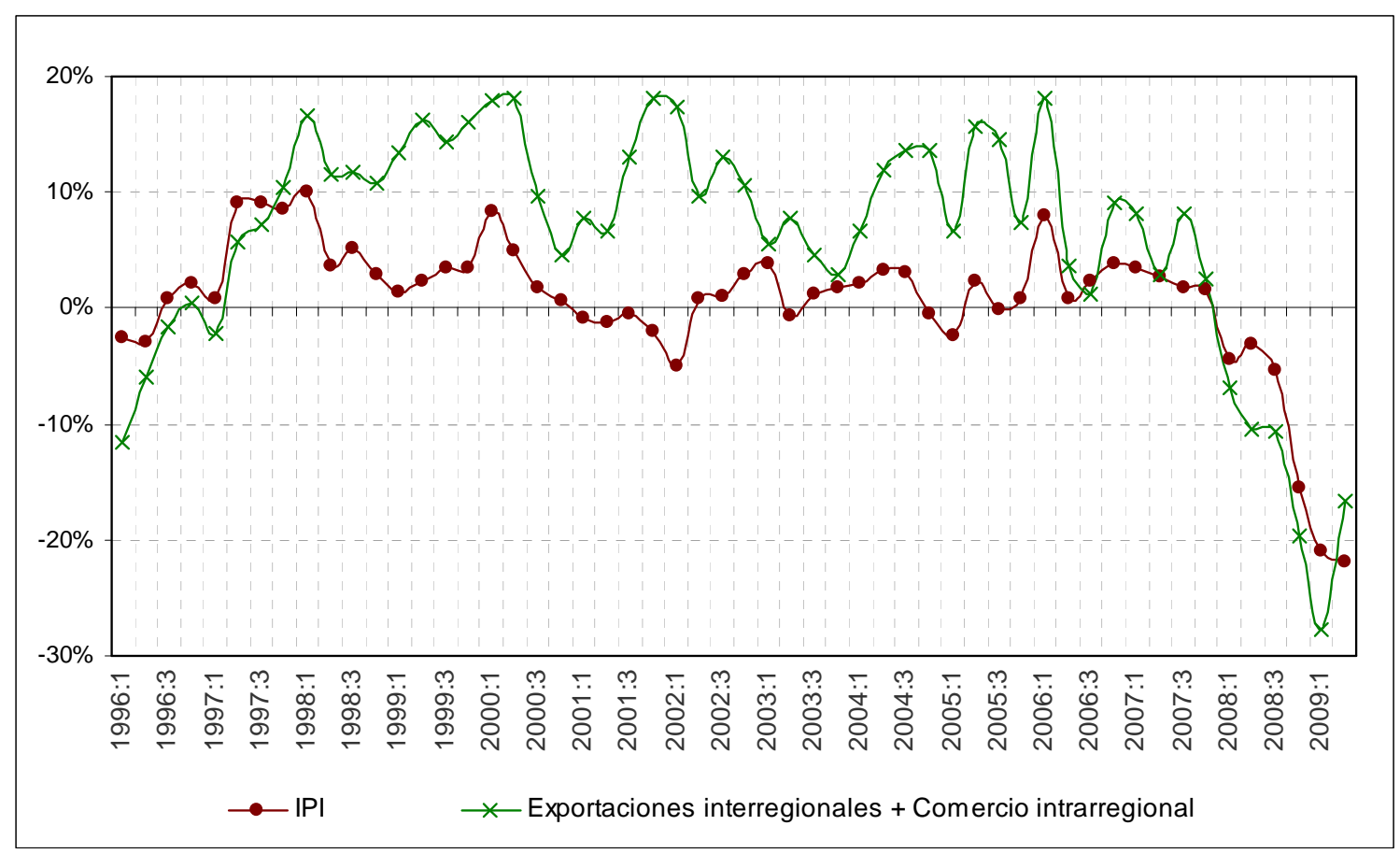

Con el objeto de aportar un punto de referencia adicional, se han comparado los flujos comerciales trimestrales estimados con sus análogos del comercio internacional también medidos en unidades físicas. Así, tal y como muestra la Tabla 4, las correlaciones entre todos los vectores se encuentran entre el $74 \%$ y el $98 \%$.

Tabla 4. Correlaciones entre los flujos internacionales e interiores. 1995-2009II

\begin{tabular}{|c|c|c|c|c|}
\cline { 2 - 5 } \multicolumn{1}{c|}{} & $\begin{array}{c}\text { Export. } \\
\text { interregional }\end{array}$ & $\begin{array}{c}\text { Export. } \\
\text { Internacional }\end{array}$ & $\begin{array}{c}\text { Import. } \\
\text { Internacional }\end{array}$ & $\begin{array}{c}\text { Comercio } \\
\text { intrarregional }\end{array}$ \\
\hline $\begin{array}{c}\text { Export. } \\
\text { Interregional }\end{array}$ & 1 & 0.80 & 0.94 & 0.98 \\
\hline $\begin{array}{c}\text { Export. } \\
\text { internacional }\end{array}$ & 1 & 0.81 & 0.74 \\
\hline $\begin{array}{c}\text { Import. } \\
\text { internacional }\end{array}$ & & 1 & 0.95 \\
\hline $\begin{array}{c}\text { Comercio } \\
\text { intrarregional }\end{array}$ & & & 1 \\
\hline Fuente: elaboración propia a partir de los datos de A.E.AT. \\
\hline
\end{tabular}


Gráfico 3. Evolución del comercio de bienes según categoría geográfica.

Tasas de crecimiento interanuales a partir de los niveles en $\mathbf{T m}$.

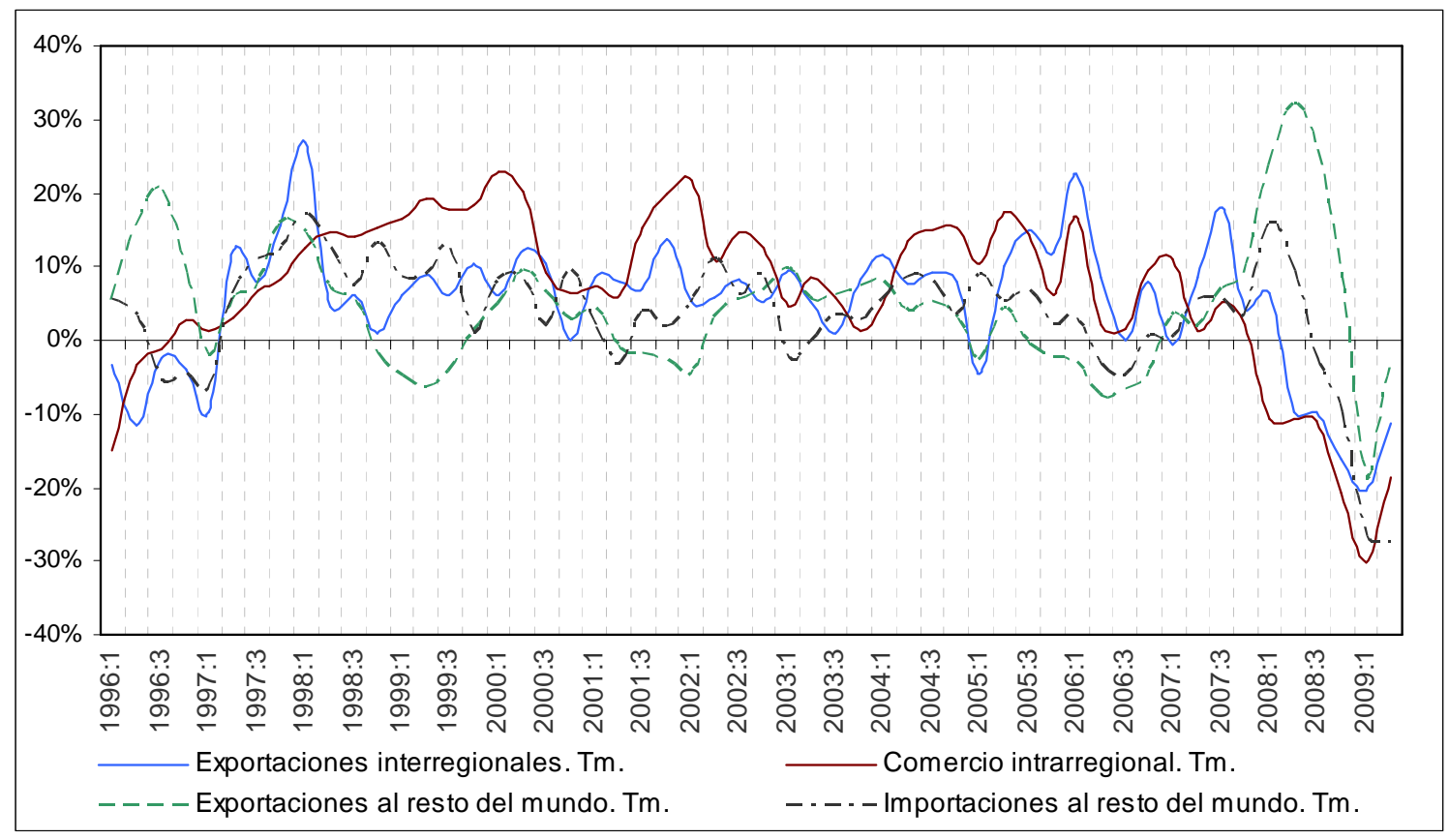

Fuente: elaboración propia a partir de datos de C-intereg y AEAT

\subsection{Capacidad predictiva del modelo}

En este epígrafe se analizan los datos obtenidos a través del proceso de extrapolación temporal (predicción) seguido para obtener datos de transporte trimestral cuando no existen datos anuales de restricción. Este proceso parte de los modelos de interpolación estimados para la trimestralización de cada uno de los tres tipos de flujos (movimientos intrarregionales más exportaciones e importaciones interregionales).

Como ya se mencionó previamente, la estimación de las series trimestrales del comercio intra y exportaciones interregionales han sido obtenidas mediante técnicas uni-variantes (Chow-Lin), mientras que las importaciones han exigido la combinación de técnicas uni-variantes y multi-variantes (Denton) $)^{8}$. En todos los casos, la estimación

\footnotetext{
${ }^{8}$ La principal diferencial entre la extrapolación y la interpolación radica en el hecho de que para la extrapolación no se dispone del dato anual de restricción de los tres tipos de flujos comerciales, por lo que los resultados de la aplicación de la metodología uni-variante no proporcionan un mismo dato anual de importaciones que de exportaciones interregionales. De acuerdo con la metodología de Denton, es necesario que exista dicha identidad entre exportaciones e importaciones interregionales. Ante esta exigencia, se han transformado los flujos de importaciones anuales de cada comunidad autónoma estimados en la Fase I, a través de su producto con el cociente que suponen las exportaciones interregionales nacionales sobre las importaciones interregionales nacionales. De esta forma, se ha logrado la identidad de las exportaciones y las importaciones anuales interregionales.
} 
de los modelos uni-variantes se ha centrado en el periodo 1995-2007 con datos anuales de restricción. Así, la extrapolación temporal (predicción) se ha realizado para el periodo 2008.I-2009.II, obteniendo un dato adelantado para nueve trimestres, permitiendo la obtención de los flujos totales de transporte de mercancías para un año completo (2008) con más de un año de antelación a la fecha en la que estarían disponibles mediante el procedimiento habitual de estimación de la base de datos anual de C-intereg.

Con vistas a la valoración de la capacidad de predicción de los modelos en aquellos periodos en los que no hay dato anual de restricción, se ha procedido a reestimar todos los modelos de trimestralización excluyendo el año 2007. De esta manera, se ha obtenido una predicción de datos trimestrales para el 2007, que una vez anualizados, pueden ser comparados con los datos efectivos del 2007, que fueron estimados por el procedimiento completo (estimación abajo-arriba habitualmente utilizado en C-intereg). A través de dicha comparación, se puede valorar la capacidad predictiva de los modelos en un año conocido (2007), asumiendo un comportamiento similar para aquellos años (y para cada trimestre) para los que no se tiene punto de referencia (2008 y 2009).

\subsection{Análisis gráfico de las predicciones del comercio interregional del 2007}

En este epígrafe se muestran los resultados de lo que ha sido un doble ejercicio de predicción para el año 2007, año para el que se cuenta con datos "efectivos" de referencia. Por un lado, se han comparado los datos anuales del 2007 obtenidos mediante la predicción basada en los modelos de trimestralización estimados para el periodo 1995-2006 (sin incluir los datos anuales del 2007 como restricción temporal) con los "datos efectivos" del 2007 (datos anuales de C-intereg). Tal y como se aprecia en los Gráfico 4, 5 y 6, los niveles de comercio obtenidos a través de la predicción para el año 2007 resultan muy similares a los valores efectivos obtenidos en la estimación desde abajo. Con ello, cabe suponer que la predicción de los datos anuales adelantados para el conjunto y para cada una de las comunidades puede ser bastante similar al dato que se obtendrá una vez que la estimación abajo-arriba esté disponible. 
Adicionalmente, se comparan los datos trimestrales obtenidos para el 2007 mediante el ejercicio de predicción basado en los modelos de trimestralización estimados para el periodo 1995-2006 (excluidos los datos anuales del 2007 como restricción) y la serie trimestral que se obtiene en el ejercicio de interpolación cuando los modelos se estiman incluyendo el 2007. Como se puede comprobar en los Gráficos 7, 8 y 9, para cada año y comunidad, los niveles obtenidos a través de la predicción resultan muy similares a los obtenidos mediante la interpolación hasta el 2007. Este hecho apunta hacia un buen comportamiento de los modelos a la hora de predecir el dato trimestral cuando no existe restricción.

Gráfico 4 Valor efectivo y predicción de las exportaciones interregionales. Miles de Toneladas. Año 2007.

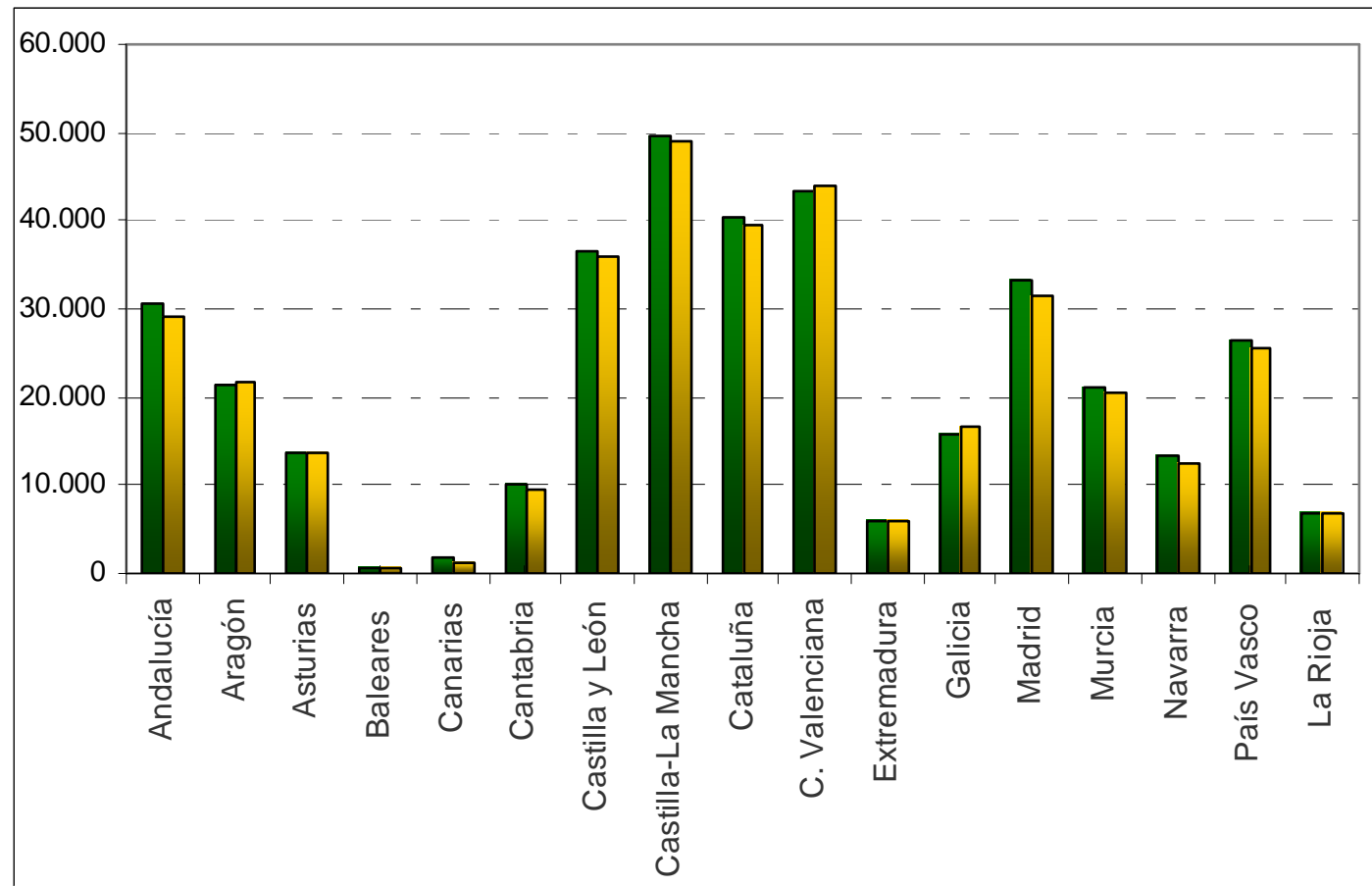

Exportaciones interregionales efectivas. Tm. $\square$ Exportaciones interregionales predicción. Tm. 
Gráfico 5: Valor efectivo y predicción de las importaciones interregionales. Miles de Toneladas. Año 2007.

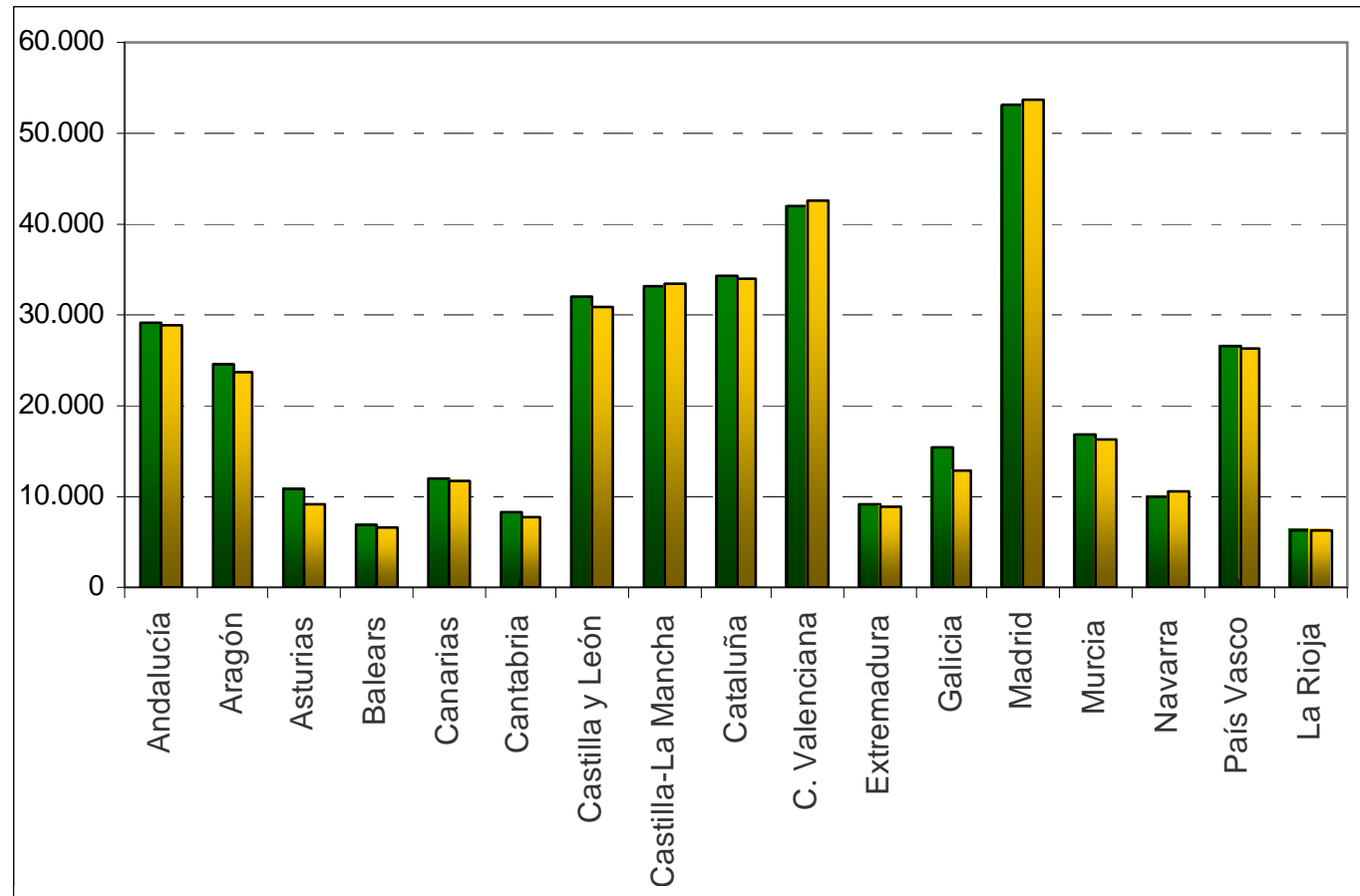

$\square$ Importaciones interregionales efectivas. Tm. $\square$ Importaciones interregionales predicción. Tm.

Gráfico 6: Valor efectivo y predicción del comercio intrarregional. Miles de Toneladas. Año 2007.

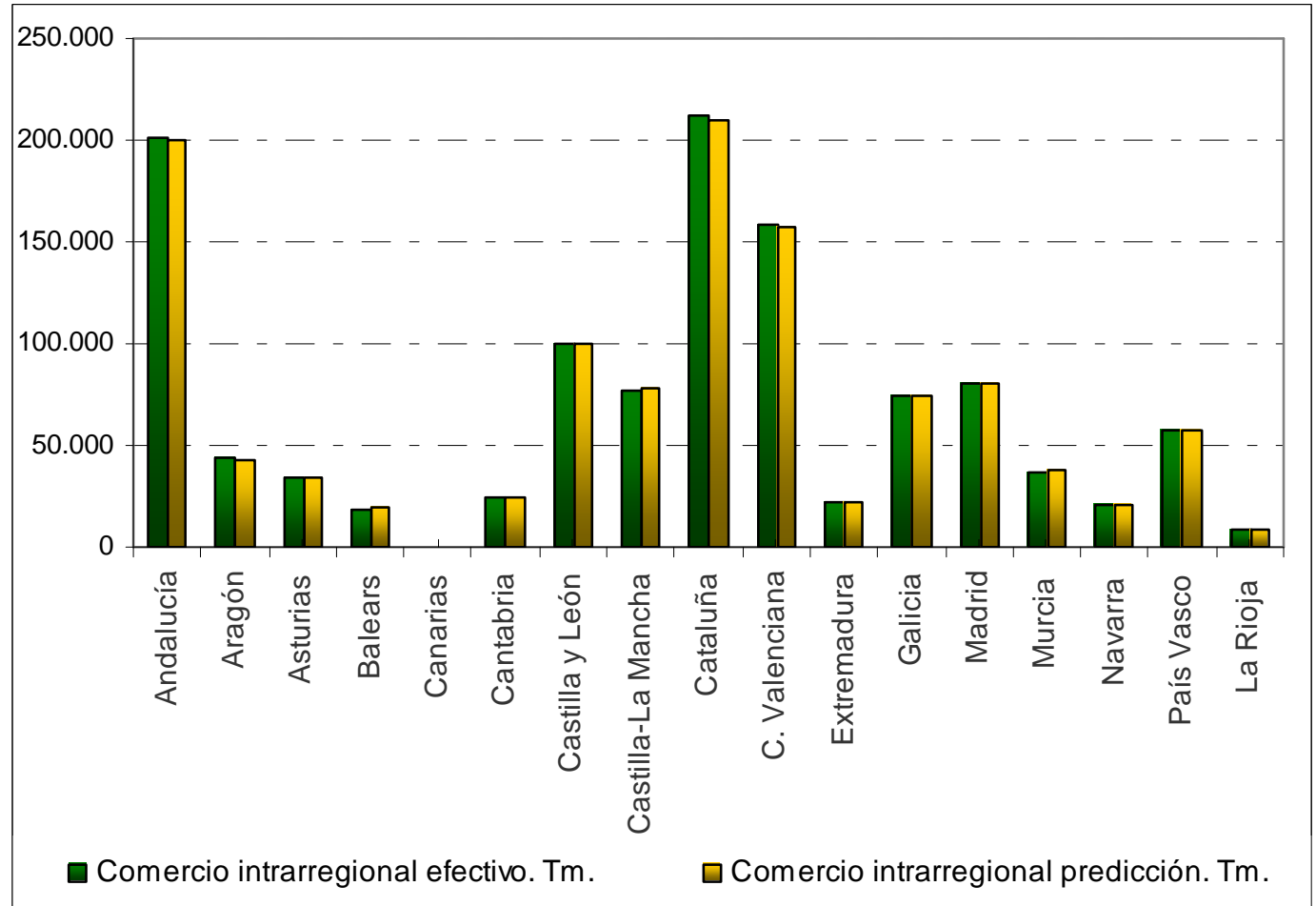


Gráfico 7: Valor de interpolación y de predicción de las exportaciones interregionales. Miles de Toneladas. 2007.

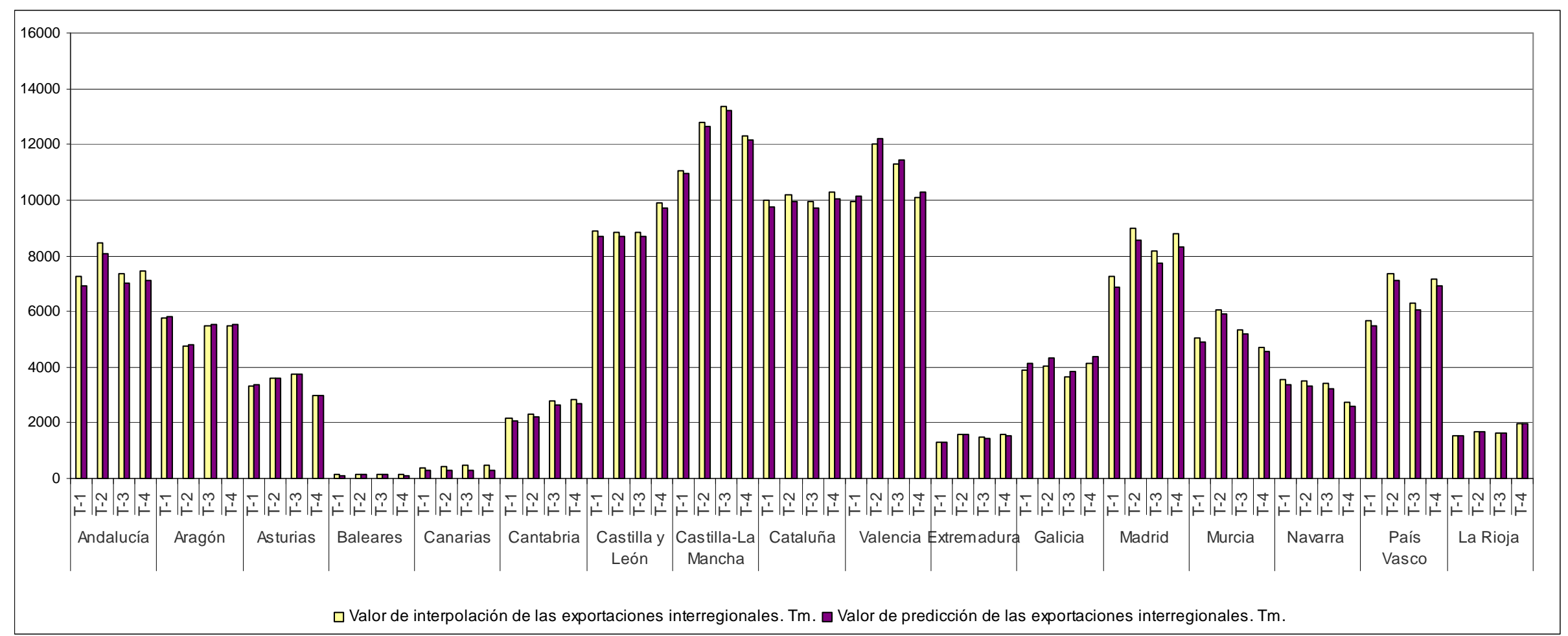


Gráfico 8: Valor de interpolación y de predicción de las importaciones interregionales. Miles de Toneladas. 2007.

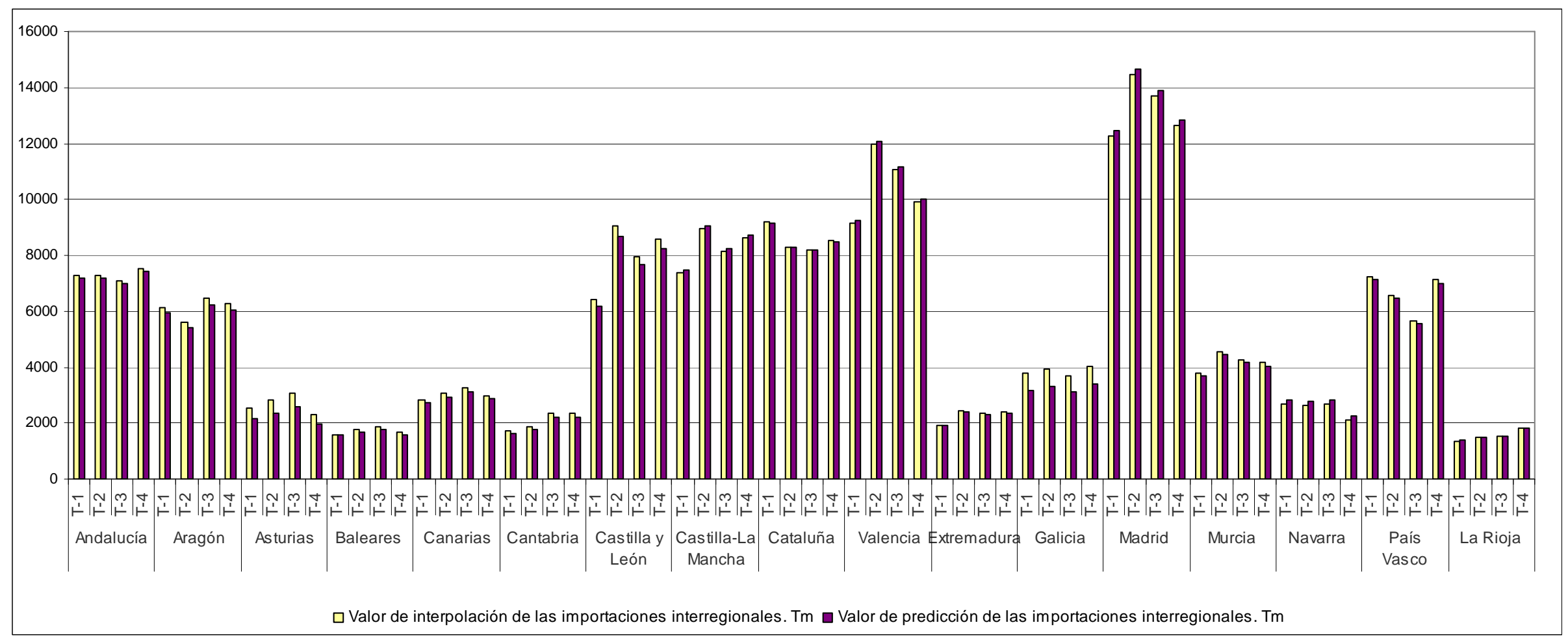


Gráfico 9: Valor de interpolación y de predicción del comercio intrarregional. Miles de Toneladas. 2007.

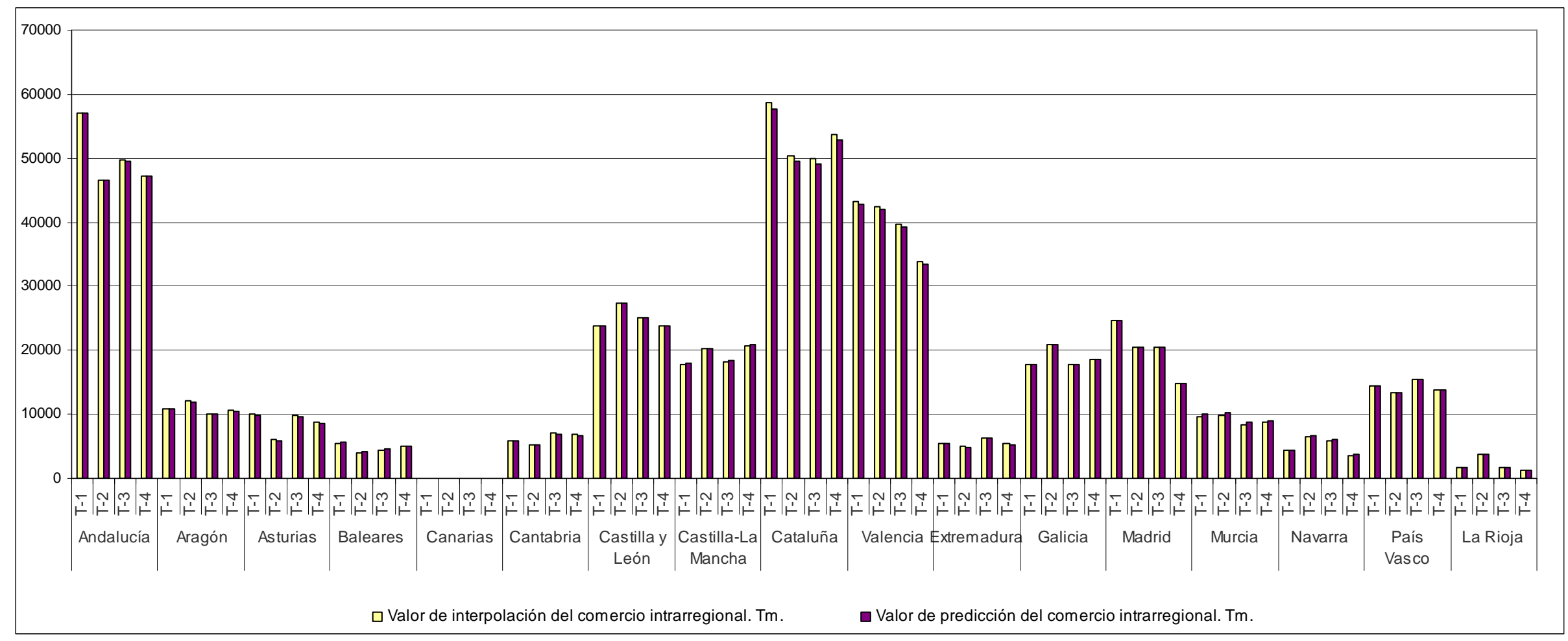




\subsection{Evaluación de la predicción del dato nacional del 2007, mediante MAPE}

Finalmente, con el objeto de evaluar cuantitativamente la predicción obtenida para el 2007 se analiza el dato nacional para los tres tipos de flujos, utilizando el error absoluto medio sobre el valor efectivo (MAPE), expresado matemáticamente de la siguiente manera:

$$
M A P E=\frac{1}{N} \sum_{i=1}^{N}\left|\frac{y_{i}-\hat{y}_{i}}{y_{i}}\right|
$$

Donde $\hat{y}$ es el valor anual de predicción para el 2007 para la comunidad autónoma i, é $y$ es el valor efectivo de ese mismo año.

Tabla 5. Evaluación de la predicción del dato nacional del 2007, mediante MAPE.

\begin{tabular}{|l|c|}
\hline \multicolumn{1}{|c|}{ Toneladas } & MAPE \\
\hline Intrarregional & $2 \%$ \\
\hline Exportaciones & $7 \%$ \\
\hline Importaciones & $5 \%$ \\
\hline
\end{tabular}

Los resultados del MAPE (Tabla 5), muestran como el error de la predicción ha sido relativamente reducido para todos los flujos, tal y como anunciaban los gráficos.

\section{Conclusiones}

El presente artículo describe una metodología original para estimar una serie amplia de nuevos indicadores sobre el transporte de mercancías intra e interregional español a nivel trimestral. Para ello, partiendo de los datos anuales de la base de datos Cintereg, se han estimado los flujos de transporte intrarregionales y los interregionales emitidos y recibidos en unidades físicas (toneladas) por cada comunidad autónoma. La metodología utilizada arranca de las experiencias previas sobre estimación de magnitudes trimestrales en España, haciendo uso de los métodos de interpolación 
temporal de carácter uni-variante y multi-variante más comunes en la literatura, basados en indicadores (Chow-Lin, 1971; Denton, 1971).

La metodología utilizada, consistente en una estimación en dos fases, ha permitido aprovechar la capacidad de predicción del método de Chow-Lin con la imposición de la restricción transversal del método de Denton para el caso de las importaciones interregionales. Hasta donde sabemos, se trataría de la primera aplicación de estas técnicas al problema de estimación de flujos de transporte. Así mismo, otra aportación interesante de este trabajo radica en la utilización de indicadores de movimiento de mercancías de los modos de transporte más relevantes para cada comunidad, sin acudir a los indicadores sobre producción, empleo o consumo.

Desde el punto de vista de los resultados, se ha obtenido una nueva colección de indicadores trimestrales para el seguimiento de la economía regional en general, y de los movimientos de mercancías dentro de España en particular. Así mismo, mediante la agregación de los flujos trimestrales obtenidos se ha podido adelantar el dato estructural de 2008 en más de un año. Más aún, la metodología aplicada ha permitido obtener predicciones "sin restricción" de las series comerciales trimestrales para el periodo 2008.I-2009.II. Finalmente, mediante el cálculo del error medio de una predicción obtenida para los flujos del 2007, se ha corroborado un buen ajuste entre la predicción y el valor efectivo para la mayor parte de las Comunidades Autónomas.

Entre los planes de mejora, se encuentra el desarrollo de métodos más compactos y eficientes para lograr la interpolación y extrapolación de las series de comercio asegurando la doble restricción, longitudinal y transversal, sin imponer una preponderancia inicial de los datos de exportaciones sobre los de importaciones. Así mismo, se contempla la extensión de este tipo de procedimientos a la trimestralización de los datos sectoriales y de comercio bilateral, ambos disponibles a nivel anual en la base de datos de C-Intereg. 


\section{Referencias bibliográficas}

Abad A. M; E.M. Quilis (2004): "Una interface para la desagregación temporal de series económicas". Boletín Trimestral de Coyuntura, n. 92 Junio.Instituto Nacional de Estadistica www.ine.es/daco/daco42/daco4214/cbtc47.pdf

Anderson, J. E. y E. Van Wincoop (2003), "Gravity with Gravitas: A Solution to the Border Puzzle", American Economic Review, 93, 1, 170-92.

Boot, J.C.G., Feibes, W. y Lisman, J.H.C. (1967) "Further methods of derivation of quarterly figures from annual data", Applied Statistics, vol. 16, n. 1, p. 65-75.

Cabrer, B., Pavía, J.M. (1999) Estimation of $J(>1)$ quarterly time series fulfilling annual and quarterly constraints, International Advances in Economic Research 5: 339349

Chen, N. (2004), "Intra-national versus International Trade in the European Union: Why Do National Borders Matter?", Journal of International Economics, 63, 1, 93-118.

Chow, G. y Lin, A.L. (1971) "Best linear unbiased distribution and extrapolation of economic time series by related series", Review of Economic and Statistics, vol. 53, n. 4, p. 372- 375 .

Denton, F.T. (1971): “Adjustment of Monthly or Quarterly Series to Annual Totals: An Approach Based on Quadratic Minimization", Journal of American Statistical Association, vol. 66, $\mathrm{n}^{\mathrm{o}}$ 333, marzo, pp. 99-102.

Di Fonzo, T. (1990) "The estimation of M disaggregate time series when contemporaneous and temporal aggregates are known", Review of Economic and Statistics, vol. 72, p. 178- 182.

Di Fonzo, T. (1994) "Temporal disaggregation of a system of time series when the aggregate is known", INSEE-Eurostat Workshop on Quarterly National Accounts, París, diciembre.

Fernández, R.B. (1981). A methodological note on the estimation of time series, The Review of Economics and Statistics 53: 471-478

Ghemawat, P., Llano C., Requena, F. (2009): "Rethinking Regional Competitiveness: Catalonia's International and Interregional Trade, 1995-2006”. IESE Business School Working Paper No. 802.

Gil, S., Llorca, R., Martínez, J.A. Y OLIVER, J. (2006), “The Border Effect in Spain”, The World Economy, 28, 1617-1631

Helliwell, J. F. (1996), “Do National Borders Matter for Quebec's Trade?”, Canadian Journal of Economics, 29, 3, 507-22.

INE. 1993. Contabilidad nacional trimestral de España: Metodología y serie trimestral 1979-1992. Instituto Nacional de Estadística: Madrid.

Litterman, R.B. (1983): “A Random Walk, Markov Model for the Distribution of Time Series”, Journal of Business and Economic Statistics, vol. 1, $\mathrm{n}^{\circ}$ 2, abril, pp. 169173.

Llano, C (2004a): "Economía espacial y sectorial: el comercio interregional en el marco Input-Output". Instituto de Estudios Fiscales. Ministerio de Economía y Hacienda. Investigaciones $\mathrm{N}^{\circ} 1,2004$.

Llano, C (2004b): "The interregional trade in the context of a multirregional input-output model for Spain”. Estudios de Economía Aplicada. .Vol.22-3, 2004. Art 22302.

Llano, C., ESTEBAN, A., PULIDO, A., PÉREZ, J (2009a): "Opening the interregional trade "black box": the C-intereg database for the Spanish economy (1995-2005)". International Regional Science Review. Under review. 
Llano C., Esteban, A; Pérez, J.; Pulido, A. (2009b): "Metodología de estimación de la base de datos C-intereg sobre el comercio interregional de bienes en España (199505)", Ekonomiaz.n ${ }^{\circ}$ 69: 245-267.

Matea, M.L. y Regil, A.V. (1994): "Métodos para la extracción de señales y para la trimestralización”, Banco de España, Documento de Trabajo n ${ }^{\circ}$ 9415, Madrid.

Mccallum, J. (1995), "National Borders Matter: Canadian-U.S. Regional Trade Patterns", American Economic Review, 85, 3, 615-23.

Minondo, A. (2003), "Comercio internacional y efecto frontera en el País Vasco", Revista de Economía Aplicada, 11 (32), 115-134.

Okubo, T. (2004), "The Border Effect in the Japanese Market: A Gravity Model Analysis", The Japanese and International Economies, 18, 1-11.

OECD (1996) Quaterly National Accounts. Sources and Methods used by OECD Member Countries. OECD, Paris, France

Oliver, J. (1997), La balança comercial amb la resta de l'Estat', en La Balança de Pagaments de Catalunya: Una aproximació als fluxos economòmics amb la resta dÉspanya i l`estranger (1993-1994). Institut d'Estudis Autonòmics, Generalitat de Catalunya, pp. 17-75.

Oliver, J., Luria J, Roca A., Pérez J. (2003), en "La apertura exterior de las regiones en España: Evolución del comercio interregional e internacional de las Comunidades Autónomas. 1995-1998". Institu dÉstudis Autonòmics. Generalitat de Catalunya. Ed Tirant lo blanch. Valencia.

Pavía J.M., Cabrer-Borrás B. (2007): On Estimating Contemporaneous Quarterly Regional GDP. Journal of Forecasting. 26, 155-170

Pavía JM, Vila LE, Escuder R. (2003a). On the performance of the Chow-Lin procedure for quarterly interpolation of annual data: some Monte-Carlo analysis. Spanish Economic Review 5: 291-305.

Pavía J.M., Vila-Ladosa L.E., Escuder Vall'Es R. (2003b): On the performance of the Chow-Lin procedure for quarterly interpolation of annual data: Some Monte-Carlo analysis. Spanish Economic Review 5, 291-305 (2003)

Pavía, J.M. (2000) Desagregación Conjunta de Series Anuales: Perturbaciones AR(1) Multivariante. Investigaciones Económicas XXIV: 727-737

Pons, E.; Pons, J. Y Suriñach, J. (1997): "Trimestralización y conciliación de magnitudes económicas: una ampliación del método de Chow-Lin", Documents de Treball de la Divisió de Ciències Jurídiques Econòmiques i Socials, E97/20, Universitat de Barcelona.

Quilis, E.M. (2001a) Sobre el método de desagregación temporal de Litterman. Instituto Nacional de Estadística. Boletín Trimestral de Coyuntura, n. 81. Septiembre, 2001

Quilis, E.M. (2001b) "Notas sobre desagregación temporal de series económicas", Instituto de Estudios Fiscales, Papeles de Trabajo n. 1/01.

Requena, F.; Llano C. (2009), "The border effect in Spain: an industry analysis", Empirica. En revisión.

Rossi, N. (1982) "A note on the estimation of disaggregate time series when the aggregate is known", Review of Economics and Statistics, vol. 64, n. 4, p. 695-696.

Santos Silva, J.M.C. y Cardoso, F. (2001) "The Chow-Lin method using dynamic models", Economic Modelling, vol. 18, p. 269-280.

Sanz, R. (1981): "Métodos de desagregación temporal de series económicas", Banco de España, Documento de Trabajo nº 22, Madrid. 
Cavero, J., Fernández-Abascal, H., Gómez, I., Lorenzo, C., Rodríguez, B., Rojo, J.L., Sanz, J.A. (1994) Hacia un modelo trimestral de predicción de la economía castellano-leonesa: El modelo Hispalink CyL. Cuadernos Aragoneses de Economía 4: $317-343$ 


\section{Anexos}

\subsection{Breve descripción de los datos anuales de C-intereg $^{9}$}

Actualmente, la base de datos de C-intereg cubre el periodo 1995-2007, con detalle a 30 ramas, 6 modos de transporte (carretera, ferrocarril, barco, avión, oleoducto y red eléctrica) en unidades físicas (Tm.) y monetarias (€). Los flujos objeto de trimestralización son los correspondientes a los flujos comercio intra e interregional total anual de bienes de las ramas $\mathrm{R} 1$ a 15 de la base de datos $\mathrm{C}$-intereg en unidades físicas (Tm.). La estimación de los flujos anuales en unidades físicas de la base de datos de CIntereg se apoya fundamentalmente sobre la utilización de las estadísticas de flujos de mercancías según los distintos modos de transporte tratadas con la mayor desagregación posible. Sintéticamente, el tratamiento de cada uno de los modos de transporte es el siguiente:

- Carretera: por el lado de los flujos de mercancías por carretera se han utilizado los ficheros facilitados por la Subdirección General de Estadística y Estudios del Ministerio de Fomento donde se recogen todos los flujos de la Encuesta Permanente de Mercancías por Carretera (EPTMC) del periodo 1995-2007 que tuvieran como punto de origen o destino un municipio español (sin incluir los flujos intra-municipales).

- Ferrocarril: se ha utilizado la información de base facilitada por el Departamento de Estadística de RENFE en relación a los flujos de transporte nacional registrados en la unidad de carga y transporte combinado, desglosados según la clasificación propia que utiliza dicha compañía (40 tipos de productos, bastante compatible con la clasificación NST/R de carretera).

- Barco: Ante la inexistencia de una matriz completa de intercambios de mercancías en régimen de cabotaje para cada tipo de producto y año del periodo 1995-2006 ha obligado a estimarla por métodos indirectos a partir de la última colección de matrices origen/destino de cabotaje publicada por Puertos del Estado (1989) y del conocimiento del volumen de cargas y descargas por puerto

\footnotetext{
${ }^{9}$ Para un análisis más detallado de la metodología se puede consultar Llano et al (2008, 2009a y 2009b)
} 
de origen/destino, por tipos de mercancías y categoría de operación (cabotaje o internacional) que se recoge en los anuarios de Puertos del Estado (1995-2006).

- Avión: se han utilizado las matrices de movimientos de mercancías entre los aeropuertos nacionales (Fuente: Dirección General de Aviación Civil. Ministerio de Fomento).

\subsection{Detalle de la trimestralización de los movimientos de mercancías (Tm.)}

Tabla 6 Correlaciones de la variable objetivo y los indicadores anualizados.

\begin{tabular}{|l|c|c|c|c|c|c|c|c|}
\hline & Andalucía & Aragón & $\begin{array}{c}\text { Castilla y } \\
\text { León }\end{array}$ & $\begin{array}{c}\text { Castilla-La } \\
\text { Mancha }\end{array}$ & Cataluña & Valencia & Madrid & Murcia \\
\hline Exportaciones & $98,4 \%$ & $99,8 \%$ & $99,9 \%$ & $99,9 \%$ & $99,4 \%$ & $99,0 \%$ & $99,3 \%$ & $99,4 \%$ \\
\hline Importaciones & $99,4 \%$ & $99,2 \%$ & $99,2 \%$ & $99,8 \%$ & $99,2 \%$ & $99,5 \%$ & $99,8 \%$ & $99,3 \%$ \\
\hline Intrarregional & $99,9 \%$ & $99,9 \%$ & $99,9 \%$ & $100,0 \%$ & $99,9 \%$ & $99,9 \%$ & $100,0 \%$ & $99,9 \%$ \\
\hline
\end{tabular}

Tabla 7 Correlaciones de la variable objetivo y los indicadores anualizados.

\begin{tabular}{|l|c|c|c|c|c|c|c|c|}
\hline & Asturias & Cantabria & Extremadura & Galicia & Navarra & La Rioja & Baleares & Canarias \\
\hline Exportaciones & $99,4 \%$ & $98,1 \%$ & $99,8 \%$ & $98,8 \%$ & $91,5 \%$ & $97,1 \%$ & $88,3 \%$ & $47,6 \%$ \\
\hline Importaciones & $98,0 \%$ & $98,9 \%$ & $99,1 \%$ & $97,7 \%$ & $89,5 \%$ & $98,7 \%$ & $86,5 \%$ & $94,1 \%$ \\
\hline Intrarregional & $99,9 \%$ & $99,9 \%$ & $99,8 \%$ & $99,6 \%$ & $93,4 \%$ & $99,9 \%$ & $99,4 \%$ & $87,0 \%$ \\
\hline Fuente: elaboración propia. \\
$\begin{array}{l}\text { Nota: Las correlaciones relativas a Baleares y Canarias se refieren a los indicadores propios de dichas comunidades. En los demás } \\
\text { casos, corresponden a los indicadores derivados de "tráfico" tomados de la EPTMC. }\end{array}$
\end{tabular}


Tabla 8 Resultados de las regresiones entre los flujos y los indicadores anuales en Tm.

MCO.

\begin{tabular}{|c|c|c|c|}
\hline \multicolumn{4}{|c|}{ Coeficiente de determinación del modelo en baja frecuencia 1995-2007 } \\
\hline & Exportaciones & Importaciones & Intrarregional \\
\hline Andalucía & $97 \%$ & $99 \%$ & $100 \%$ \\
\hline Aragón & $100 \%$ & $99 \%$ & $100 \%$ \\
\hline Asturias & $99 \%$ & $90 \%$ & $100 \%$ \\
\hline Baleares & $80 \%$ & $79 \%$ & $99 \%$ \\
\hline Canarias & $22 \%$ & $93 \%$ & $82 \%$ \\
\hline Cantabria & $97 \%$ & $98 \%$ & $100 \%$ \\
\hline Castilla y León & $100 \%$ & $99 \%$ & $100 \%$ \\
\hline Castilla La Mancha & $100 \%$ & $100 \%$ & $100 \%$ \\
\hline Cataluña & $99 \%$ & $100 \%$ & $100 \%$ \\
\hline C. Valenciana & $99 \%$ & $99 \%$ & $100 \%$ \\
\hline Extremadura & $100 \%$ & $99 \%$ & $100 \%$ \\
\hline Galicia & $98 \%$ & $87 \%$ & $99 \%$ \\
\hline Madrid & $99 \%$ & $100 \%$ & $100 \%$ \\
\hline Murcia & $99 \%$ & $98 \%$ & $100 \%$ \\
\hline Navarra & $99 \%$ & $99 \%$ & $100 \%$ \\
\hline País Vasco & $99 \%$ & $99 \%$ & $100 \%$ \\
\hline La Rioja & $96 \%$ & $99 \%$ & $100 \%$ \\
\hline & & & \\
\hline
\end{tabular}

Tabla 9. Correlogramas de regiones cuyo Durbin Watson plantea problemas de autocorrelación

Flujo de exportaciones inter-regionales Tm.

\section{Canarias **}

Date: 11/03/09 Time: 11:02

Date: 11/03/09 Time: 11
Sample: 19952007
Included observations: 13

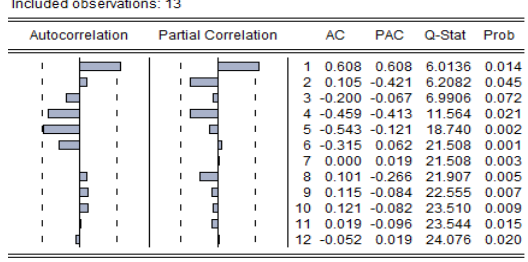

Cantabria *

Date: 11/03/09 Time: $11: 04$
Sample: 1995 2007
Included observations: 13

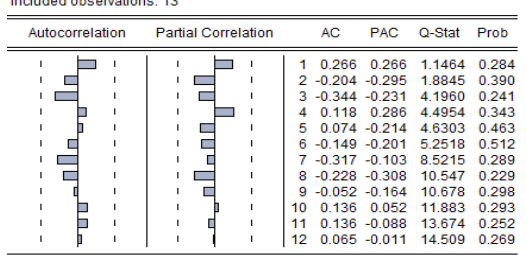

Flujo de importaciones inter-regionales Tm.

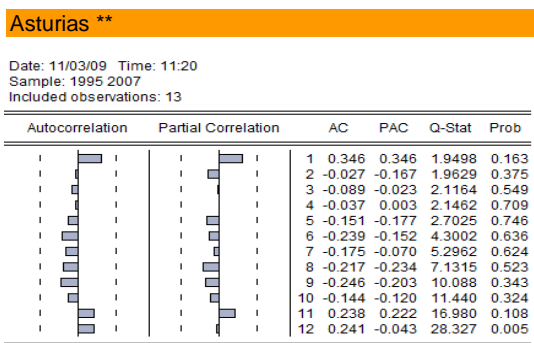

\section{Baleares **}

Date: 11/03/09 Time: 11:25

Sample: 19952007
Included observations: 13

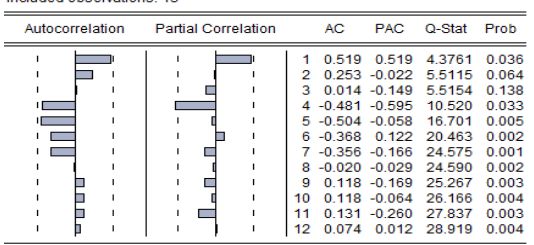

Flujo de comercio intra-regional Tm.

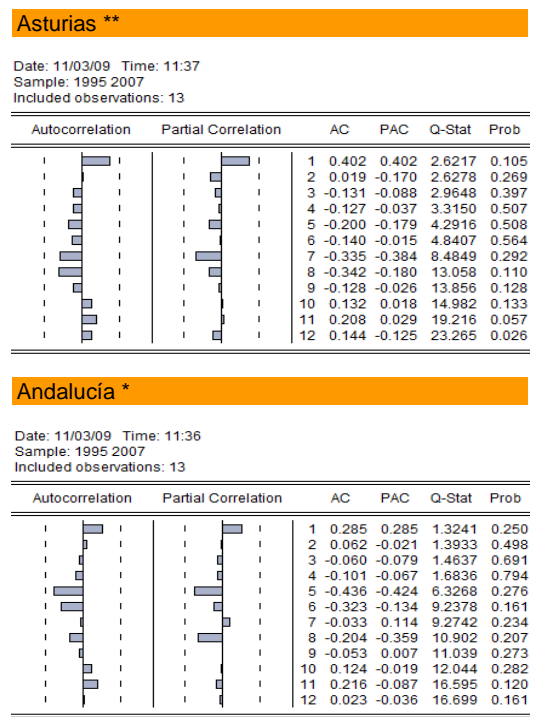


Flujo de exportaciones inter-regionales $\mathrm{Tm}$. Castilla León *

Date: 11/03/09 Time: 11:05

Sample: 19952007
Included observations: 13

\begin{tabular}{|c|c|c|c|c|c|c|}
\hline Autocorrelation & Partial Correlation & & $A C$ & PAC & Q-Stat & Prob \\
\hline 曰 & ص & & 0.321 & 0.321 & 1.6739 & \\
\hline • & & & $\begin{array}{r}0.106 \\
-0.476\end{array}$ & $\begin{array}{r}0.003 \\
-0.569\end{array}$ & $\begin{array}{l}1.8720 \\
6.2812\end{array}$ & $\begin{array}{l}0.3 \\
0.0\end{array}$ \\
\hline : 둥 & $\sigma^{3}$ & & $\begin{array}{l}-0.274 \\
-0.394\end{array}$ & $\begin{array}{r}0.069 \\
-0.287\end{array}$ & $\begin{array}{l}7.9076 \\
111695\end{array}$ & $\begin{array}{l}0.4 \\
0.8\end{array}$ \\
\hline & & & & -0.155 & $\begin{array}{l}11.773 \\
\end{array}$ & 0.0 \\
\hline & & & -0.018 & & $\begin{array}{l}11.784 \\
11.304\end{array}$ & \\
\hline 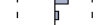 & 는 & & & & $\begin{array}{l}13.3 .04 \\
13.488\end{array}$ & ${ }_{0}^{0}$ \\
\hline & ㄷ. & & & & & \\
\hline & 미 & & -0.00 & -0.171 & 13.550 & 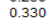 \\
\hline
\end{tabular}

\section{Castilla la Mancha *}

Date: 11/03/09 Time: 11:06

Sample: 19952007
Included observations: 13

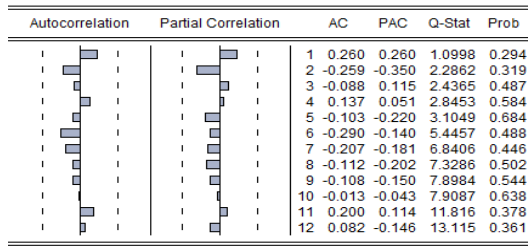

\section{Madrid}

Date: 11/03/09 Time: 11:07

Sample. 19952007

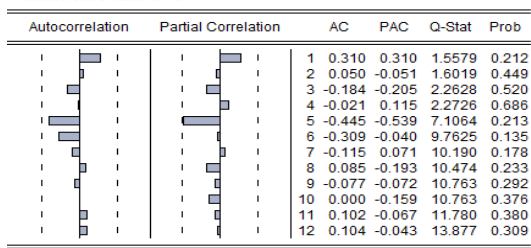

Murcia *

Date: $11 / 03 / 09$ Time: $11: 08$
Sample: 1995 2007
Included observations: 13

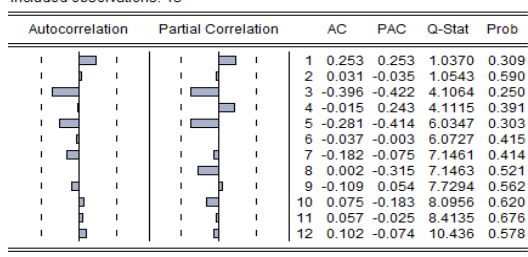

La Rioja *

Date: $11 / 03 / 09$ Time: $11: 12$
Sample: 1995 2007
Included observations: 13

\begin{tabular}{|c|c|c|c|c|c|c|}
\hline Autocorrelation & Partial Correlation & & $A C$ & PAC & Q-Stat & \\
\hline 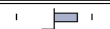 & 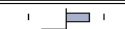 & & & & 2.0158 & \\
\hline & : & & & & & \\
\hline & 1 & & & & & \\
\hline & 5 & & & & & \\
\hline & & & & & & \\
\hline & & & & & & \\
\hline & & & & & & \\
\hline & & & -0.0 & -0.009 & 11.406 & \\
\hline
\end{tabular}

Flujo de importaciones inter-regionales Tm.

\section{Castilla León *}

Date: 11/03/09 Time: 11:28

Sample: 19952007
Included observations: 1

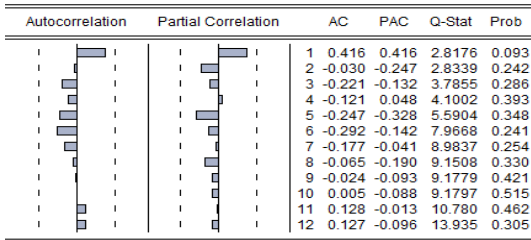

\section{Cataluña *}

Date: 11/03/09 Time: $11: 29$
Sample: 19952007

Sample: 1995 2007
Included observations: 13

\begin{tabular}{llllll}
\hline \hline Autocorrelation & Partial Correlation & AC & PAC & Q-Stat & Prob \\
\hline
\end{tabular}

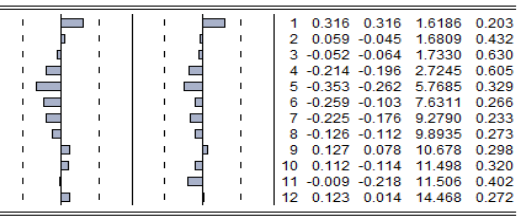

\section{Galicia **}

Date: 11/03/09 Time: 11:30

Sample: 19952007
Included observations: 13

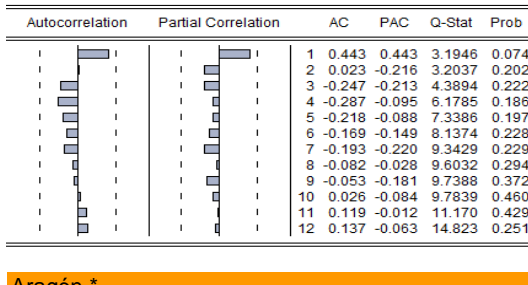

Aragón *

Date: 11/03/09 Time: 11:20

Sample: 19952007
Included observations: 13

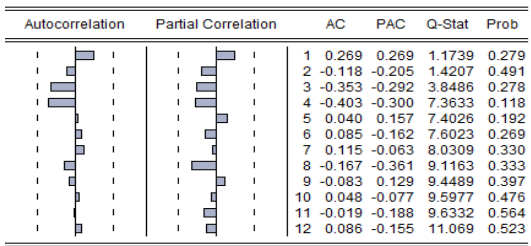

Canarias *

Date: 11/03/09 Time: 11:21

Sample: 19952007
Included observations: 13

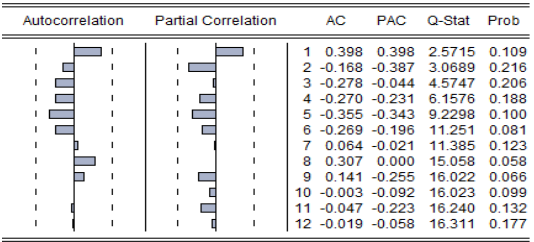

Cantabria *

Date: 11/03/09 Time: 11:26

Sample: 19952007
Included observations: 13

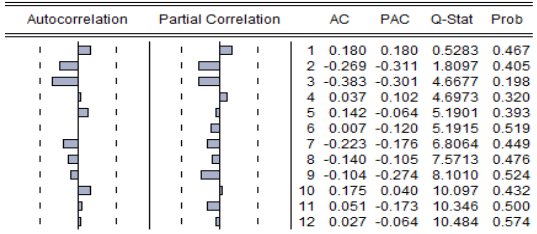

Flujo de comercio intra-regional Tm.

\section{Canarias}

Date: 11/03/09 Time: 11:30

Sample: 19952007
Included observations: 13

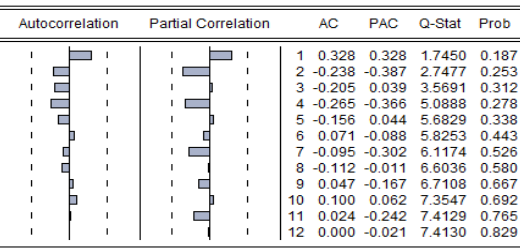

\section{Cataluña}

Date: 11/03/09 Time: 11:39

Sample: 19952007
Included observations: 13

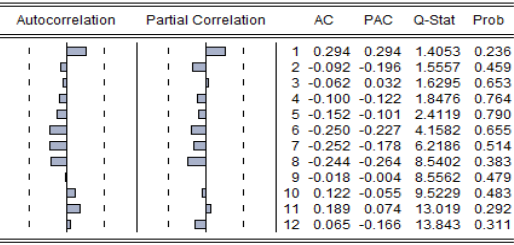

\section{Galicia}

Date: 11/03/09 Time: 11:40

Sample: 19952007

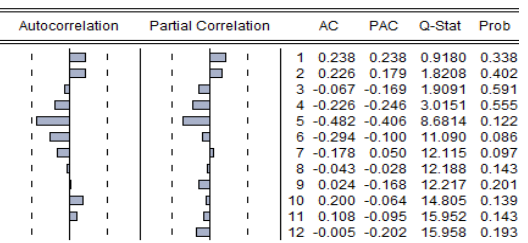

País Vasco *

Date: 11/03/09 Time: $11: 41$

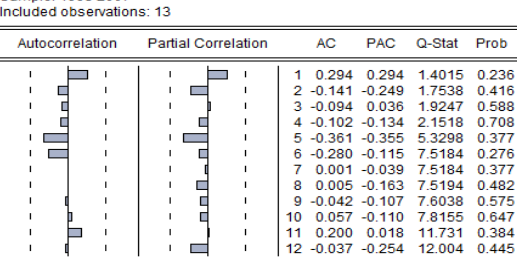

